\title{
Triple visual hemifield maps in optic chiasm hypoplasia
}

2 Khazar Ahmadi $^{1}$, Alessio Fracasso ${ }^{2,3,4,5, \dagger}$, Robert J. Puzniak ${ }^{1, \dagger}$, Andre D. Gouws ${ }^{6}$, Renat

3 Yakupov $^{7,8}$, Oliver Speck ${ }^{7,8,9,10}$, Joern Kaufmann ${ }^{11}$, Franco Pestilli ${ }^{12}$, Serge O. Dumoulin ${ }^{2,4,13}$,

4 Antony B. Morland ${ }^{6,14}$ \& Michael B. Hoffmann ${ }^{1,10, *}$

$5{ }^{1}$ Department of Ophthalmology, Otto-von-Guericke University, Magdeburg, 39120, Germany;

$6 \quad{ }^{2}$ Department of Experimental Psychology, Helmholtz Institute, Utrecht University, Utrecht, 3584

7 CS, The Netherlands; ${ }^{3}$ Department of Radiology, University Medical Center Utrecht, Utrecht,

$83584 \mathrm{CX}$, The Netherlands; ${ }^{4}$ Spinoza Centre for Neuroimaging, Amsterdam, 1105 BK , The

9 Netherlands; ${ }^{5}$ Institute of Neuroscience and Psychology, University of Glasgow, Glasgow, G12

10 8QB, UK; ${ }^{6}$ Department of Psychology, York Neuroimaging Centre, University of York, York,

11 YO10 5NY, UK; ${ }^{7}$ Department of Biomedical Magnetic Resonance, Institute for Physics, Otto-

12 von-Guericke University, Magdeburg, 39120, Germany; ${ }^{8}$ German Center for Neurodegenerative

13 Diseases, Magdeburg, 39120, Germany; ${ }^{9}$ Leibniz Institute for Neurobiology, Magdeburg, 39118,

14 Germany; ${ }^{10}$ Center for Behavioral Brain Sciences, Magdeburg, 39106, Germany; ${ }^{11}$ Department of

15 Neurology, Otto-von-Guericke-University, Magdeburg, 39120, Germany; ${ }^{12}$ Department of

16 Psychological and Brain Sciences, Indiana University, Bloomington, IN, 1101 E, USA;

$17{ }^{13}$ Department of Experimental and Applied Psychology, VU University Amsterdam, Amsterdam,

181081 BT, The Netherlands; ${ }^{14}$ Centre for Neuroscience, Hull-York Medical School, University of

19 York, York, YO10 5DD, UK

20 These authors contributed equally.

$21 *$ Correspondence:

Michael B. Hoffmann

23 Department of Ophthalmology, Visual Processing Laboratory, Leipziger Str. 44, 39120,

24 Magdeburg, Germany

Email: michael.hoffmann@med.ovgu.de 


\section{Abstract}

29 In humans, each hemisphere comprises an overlay of two visuotopic maps of the contralateral

30 visual field, one from each eye. Is the capacity of the visual cortex limited to these two maps or 31 are plastic mechanisms available to host more maps? Using an integrative approach of 32 submillimeter fMRI, diffusion-weighted imaging and population receptive field mapping, we 33 found three hemiretinal inputs to converge onto the left hemisphere in a rare individual with 34 chiasma hypoplasia. This generates extremely atypical responses in striate and extrastriate 35 cortices, specifically an overlay of three hemifield representations. Unexpectedly, the effects of 36 this large abnormality on visual function in daily life are not easily detected. We conclude that

37 developmental plasticity including the re-wiring of local intra- and cortico-cortical connections is 38 pivotal to support the coexistence and functioning of three hemifield maps within one 39 hemisphere.

40 Keywords: Diffusion imaging, fMRI, optic chiasm, plasticity, retinotopy, visual cortex 


\section{Introduction}

43 Topographic maps of the contralateral visual field are instrumental for the functionality of the

44 human visual cortex and are considered a core principle of the notion of hemispheric specialization (Huberman et al., 2008; Wandell et al., 2007). A fundamental prerequisite for the formation of these maps is the partial decussation of the optic nerves at the optic chiasm. Here the fate of axons from the eyes is decided such that axons from the nasal retina cross the midline and project to the contralateral hemisphere, while fibers from the temporal retina remain uncrossed and project ipsilaterally. As a consequence of this partial decussation, each hemisphere receives binocular input from the contralateral visual field. While acquired damage to the optic chiasm results in bitemporal hemianopia (Weber and Landau, 2013), congenital chiasma malformations leave major aspects of visual function intact (Hoffmann et al., 2007; Hoffmann and Dumoulin, 2015; Klemen et al., 2012). This renders these conditions invaluable models for the study of the foundations of visual pathway formation and the scope of its plasticity in humans.

In individuals affected with congenital chiasmatic abnormalities [absence of optic nerve crossing in achiasma and hemihydranencephaly (Apkarian et al., 1994; Fracasso et al., 2016; Hoffmann et al., 2012; Muckli et al., 2009; Victor et al., 2000) or enhanced crossing in FHONDA and albinism (Ahmadi et al., 2018; Apkarian et al., 1983; Hoffmann et al., 2003; von dem Hagen et al., 2008)], the visual cortex receives erroneous input from the ipsilateral visual field in addition to the normal input from the contralateral visual field. This results, at the macroscopic scale, in two superimposed retinotopic maps of opposing hemifields in V1 (Ahmadi et al., 2018; Bao et al., 2015; Davies-Thompson et al., 2013; Hoffmann et al., 2012, 2003; Kaule et al., 2014; Muckli et al., 2009). Remarkably, at the mesoscopic scale, these maps are interdigitated and form hemifield dominance domains (Olman et al., 2016), that are reminiscent of the ocular dominance domains in the normal visual system. It appears therefore that the reassignment of ocular dominance domains to hemifield dominance domains is a simple mechanism to accommodate two hemifield maps, either two representations of one visual hemifield via binocular input in normal vision or two representations of opposing hemifields via monocular input in congenital chiasma malformations (Hoffmann and Dumoulin, 2015).

These observations prompt the important question, whether V1 is limited to hosting two hemifield maps, or whether the scope of plasticity in human V1 allows for the accommodation of even more maps. We identified an individual with an extremely rare type of chiasma hypoplasia that allowed us to address this question. Three types of investigations were performed using 3 
74 and 7 Tesla MRI: (i) diffusion-weighted imaging (DWI) to specify the projection error of the

75 optic nerves at the optic chiasm, (ii) population receptive field (pRF) mapping (Ahmadi et al., 76 2018; Dumoulin and Wandell, 2008) to determine the cortical visual field maps, and (iii)

77 submillimeter fMRI to examine the cortical fine-grain structure. Our results demonstrate that

78 three hemifield maps can be accommodated within a single V1. We propose that mechanisms of

79 developmental plasticity that are exceeding the simple reassignment of ocular dominance

80 domains to hemifield dominance domains enable these three maps to be hosted in V1.

\section{Results}

82 Case description

83 A 26-year-old female with chiasma hypoplasia ('CHP') participated in the study. Her best84 corrected decimal visual acuity (Snellen acuity) was 0.63 (20/32) for the dominant right eye and $850.25(20 / 80)$ for the left eye. She had moderate vertical nystagmus, strabismus [alternating 86 strabismus, esotropia $\left(5^{\circ}\right)$, and vertical deviation $\left(7^{\circ}\right)$ with alternating suppression of each eye] 87 and no stereoscopic vision. Humphrey-like visual field testing (see Methods) revealed normal 88 visual fields in both eyes. Decussation anomalies were confirmed with visual evoked potentials 89 (VEPs) and T1-weighted MRI at the age of 22. She reported an otherwise normal developmental 90 and medical history and there was no family history of ophthalmological or neurological 91 disorders.

Atypical lateralization pattern revealed by submillimeter fMRI data

93 Submillimeter fMRI at 7T was used to evaluate the cortical lateralization pattern in response to 94 bilateral contrast reversing black and white checkerboards presented to each eye separately (see 95 Methods). In a neuro-typical visual system, bilateral stimulation of each eye leads to 96 bihemispheric activation (Figure S1). In CHP, however, bilateral stimulation of the left eye 97 yielded predominant responses on the ipsilateral occipital cortex i.e. on the left hemisphere, and 98 only a marginal activation was observed on the contralateral hemisphere. In contrast, 99 considerable bilateral activation was found during bilateral stimulation of the right eye, indicating 100 that part of the nasal afferents decussate at the chiasm and project to the contralateral hemisphere 101 (Figure 1). This revealed that the misrouting pattern in CHP is distinct from complete achiasma 102 where bilateral stimulation of each eye results in complete ipsilateral activation. 
A) Left eye vs rest block

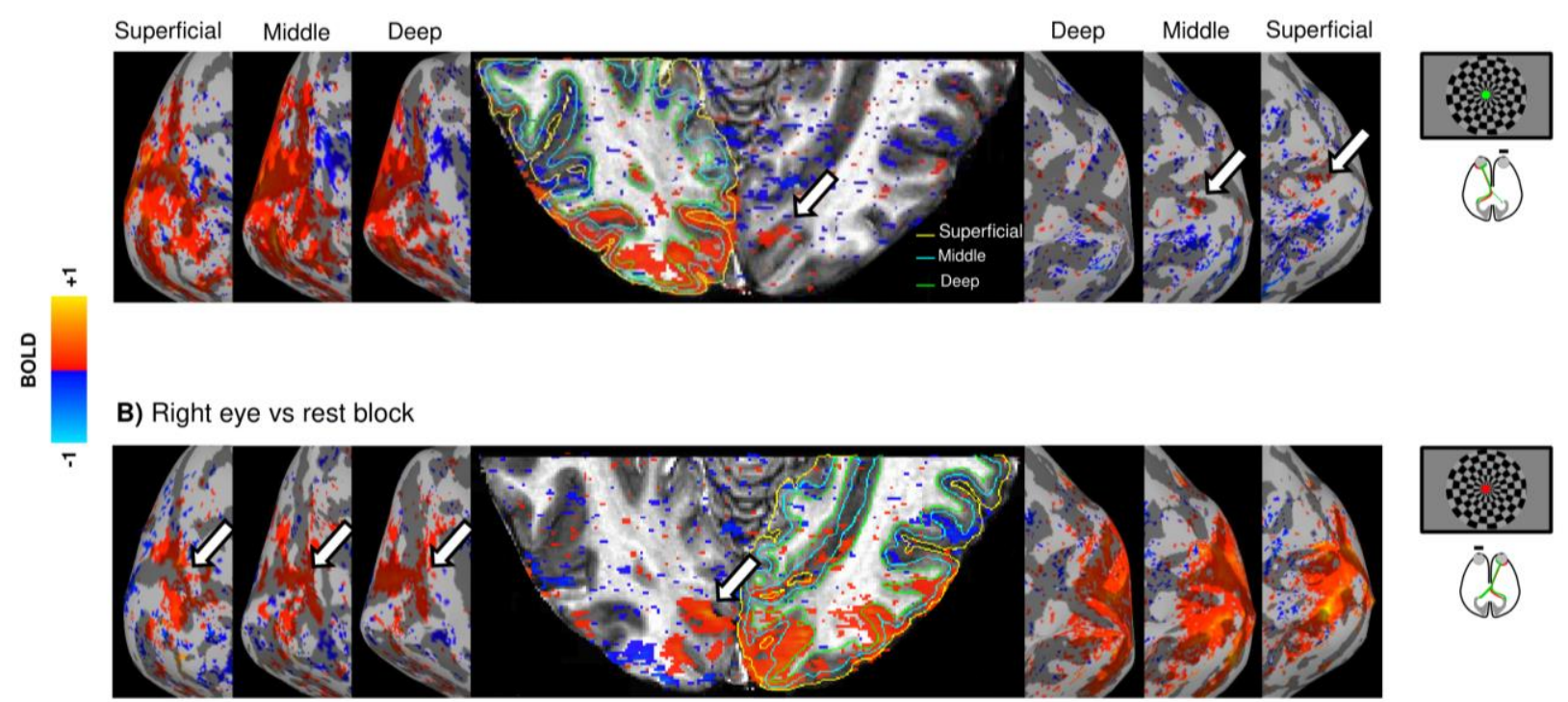

The cortical activation is projected onto a clipped anatomical image of the occipital cortex and stimulation vs rest elicits predominantly unilateral activation on the ipsilateral hemisphere with a small residual activation on the contralateral hemisphere, indicated by white arrows. B) Right eye stimulation vs rest elicits bilateral activation, i.e. on the ipsilateral hemisphere and also on part of the contralateral hemisphere (white arrows). The activation maps consist of signal amplitude expressed as the $\beta$ coefficient from the general linear model(GLM) thresholded by cluster size and mean Student's T statistic (cluster $=20$, threshold by $\mathrm{T}=1.98, \mathrm{p}=0.05$, uncorrected).

\section{Optic nerve misrouting revealed with DWI}

114 The above results predicted that the proportion of crossing fibers from the right eye would exceed

115 that from the left eye. More direct evidence for this specific misrouting of the optic nerves in 116 CHP was provided by a quantitative assessment of the streamlines at the optic chiasm based on 117 DWI data (see Methods). For CHP and 8 individuals of a control cohort, a total of four regions of 118 interest (ROIs) were selected, one in each of the two optic nerves and one in each of the two optic 119 tracts, to identify streamlines connecting each optic nerve with the (i) ipsilateral and (ii) contralateral optic tract, i.e. uncrossed and crossed projections. The proportion of the uncrossed, i.e. ipsilateral, projections was similar for the right and left optic nerves in both CHP (42\% vs $58 \%$ ) and controls (53\% vs 47\%). In contrast, the proportion of the crossed, i.e. contralateral, projections was greater for the right than for the left eye in CHP (73\% vs 27\%) but was balanced for controls (49\% vs 51\%). This underscores the asymmetric distribution of crossing afferents at the optic chiasm in CHP, which is in accordance with the above fMRI findings. A 3D rendering of the tracked streamlines is illustrated in Figure 2. 

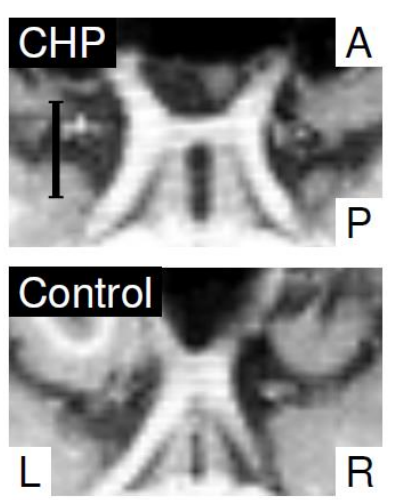

Ipsilateral streamlines
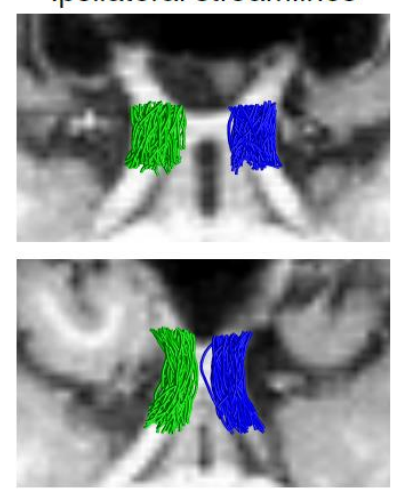
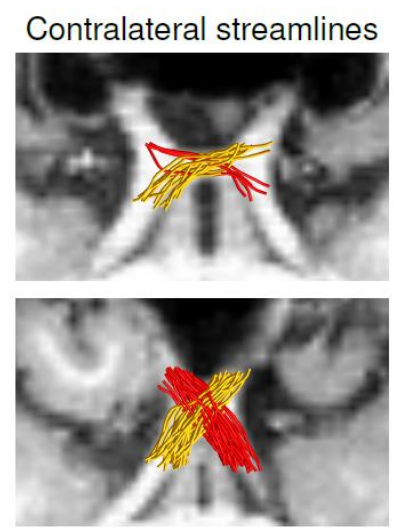

All streamlines
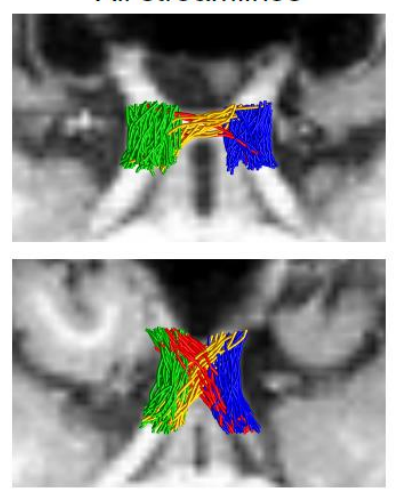

Figure 2. Tractography of the optic chiasm. Axial slices without and with tractography overlay. The scale bar represents $1 \mathrm{~cm}$. L-R and A-P stand for left-right and anterior-posterior directions, respectively. Top row) in CHP, the ipsilaterally projecting streamlines (blue and green for right and left optic nerve, respectively) are largely symmetrically distributed, while there is a predominance of contralaterally projecting streamlines for the right compared to the left optic nerve (yellow and red, respectively). Bottom row) in the control participant, both ipsi- and contralaterally projecting streamlines of the right and left optic nerves are largely symmetrically distributed. For clarity, only $0.25 \%$ of the generated streamlines are rendered.

\section{Three overlaid hemifield representations revealed by pRF mapping}

Based on the response lateralization pattern observed in the submillimeter fMRI data, we speculated that a significant part of the visual cortex on the left occipital lobe receives input from three hemiretinae, from the two hemiretinae of the ipsilateral, i.e. left, eye and from the nasal hemiretina of the contralateral, i.e. right eye. To test this hypothesis and to determine the specific mapping of the three inputs, pRF mapping (Dumoulin and Wandell, 2008) was performed during monocular stimulation of each eye and hemifield separately (see Methods). In the control participant, visuotopic maps of each hemifield were found on the contralateral hemisphere (see Figure S2). Remarkably, stimulation of the left eye in CHP revealed orderly organized eccentricity and polar angle maps of both ipsi- and contralateral hemifields on the left hemisphere across the three early visual areas (V1-V3; Figure 3 A \& B). Left and right hemifield representations were superimposed within each visual area in a mirror-symmetrical manner, in accordance with previous reports of complete achiasma (Hoffmann et al., 2012; Kaule et al., 2014). There was a small normal representation along the horizontal meridian on the contralateral, i.e. right, hemisphere. For hemifield mapping of the right eye in CHP, a similar picture was obtained, i.e. mirror-symmetrical superposition of orderly visuotopic maps of opposing hemifields (Figure $3 \mathrm{C} \& \mathrm{D}$ ). Importantly, the residual normal representation from the 
right eye was much more extensive than that from the left eye (Figure 3D), which is consistent with the above submillimeter fMRI and DWI findings. Notably, this residual normal representation appeared to be superimposed onto the other two maps from the left eye (Figure 3 A \& B). As shown in Figure 3D, the residual normal representation of the right hemifield covered a large part of V1 and spanned the entire polar angle range, from the lower vertical meridian in the dorsal portion of $\mathrm{V} 1$, through the horizontal and to the upper vertical meridian in the ventral portion of $\mathrm{V} 1$ and thus followed the normal retinotopic pattern. The observed retinotopic pattern of this residual input was not restricted to V1 and partially spread to V2 and V3. In conclusion, we found a superposition of three retinotopic representations i.e., two representations from opposing visual hemifields mediated by the left eye plus an additional representation of the contralateral hemifield from the right eye, in the left hemisphere of CHP. This is in contrast to the retinotopic organization of the neuro-typical visual system where each hemifield is represented on the contralateral hemisphere (Figure S2). A summary of this finding is provided in Figure 4 which illustrates the co-localization of three retinotopic representations in the left visual cortex of CHP. This prompted the question of the functional characteristics and the fine-structure of these maps in V1 and beyond.

\section{Responsivity of the visual cortex receiving triple hemifield input}

To compare the activation of the early visual cortex across the three hemifield-mapping conditions and to assess how the activation is propagated from V1 to V2 and V3, we determined the area of activated cortex in the early areas of the left hemisphere. As a reference, we used the condition of contralateral hemifield mapping via the left, i.e. ipsilateral, eye (normal input) for normalization and thus determined the relative activated area for both ipsilateral hemifield mapping via the left eye (abnormal input) and contralateral hemifield mapping via the right eye (residual normal input). The normal and abnormal inputs from the left eye activate a similar expanse of V1, V2 and V3. In contrast, the residual normal input from the right eye activates smaller proportions of V1, V3, and specifically V2 (Figure 5A). Subsequently, we obtained a measure of the reliability of the input for the ROIs that comprise the overlay of the three hemifield representations ( $\mathrm{ROI}_{3 \text { maps }}$ ). For this purpose, we determined the goodness of fit of the pRF model, i.e. mean variance explained (VE; Figure 5B). Although the area of cortex mapping the residual contralateral input of the right eye is smaller, the VE associated with this input does not appear to be markedly reduced compared to those driven by the normal and abnormal inputs 
the extrastriate cortex. The assessment of pRF-size properties and V1-referred connective field (CF) estimates in V2 and V3 suggest that the cortico-cortical connectivity underlying this propagation might be altered in CHP (see Figure S3).
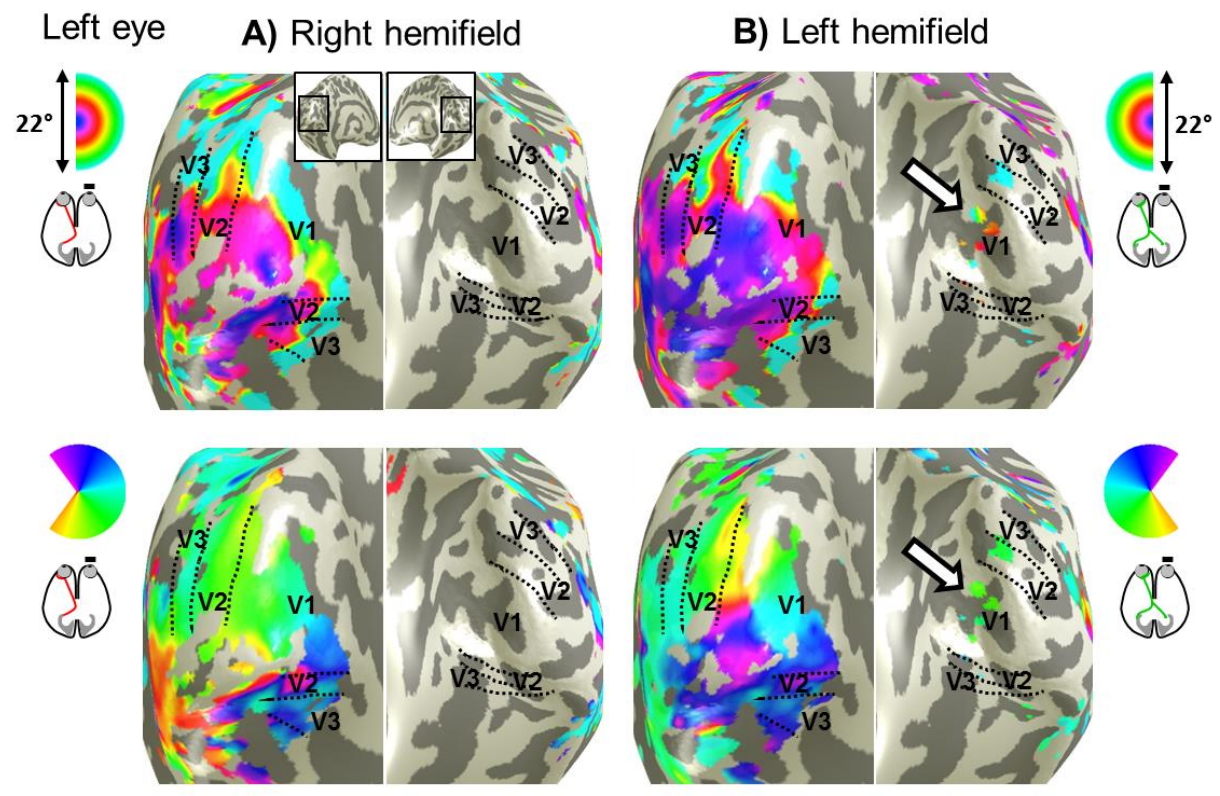

$\begin{array}{lll}\text { Right eye } & \text { C) Left hemifield }\end{array}$
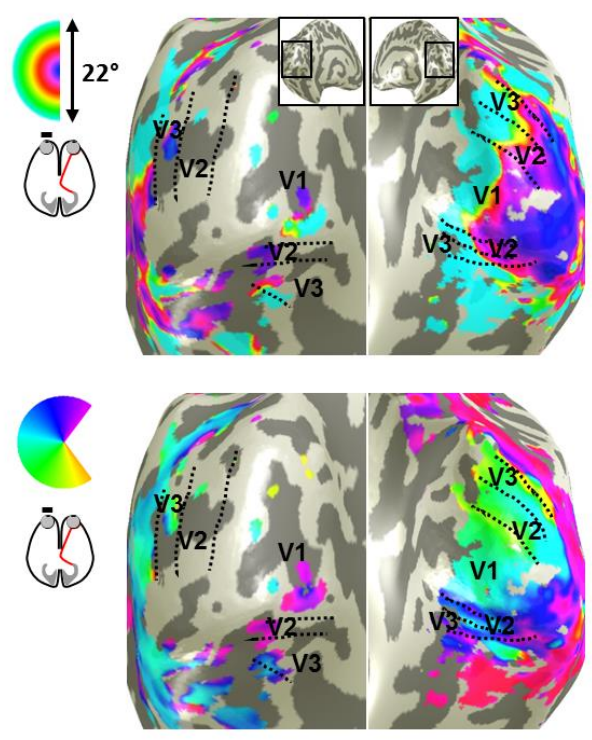
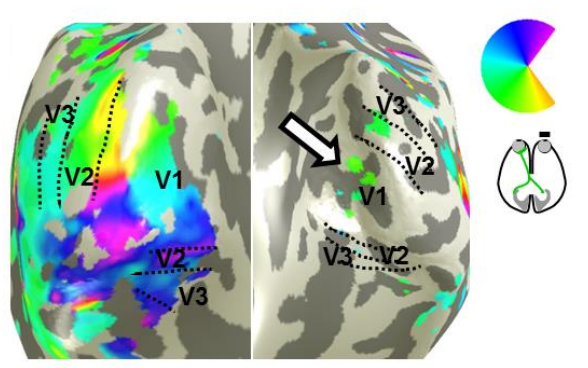

D) Right hemifield
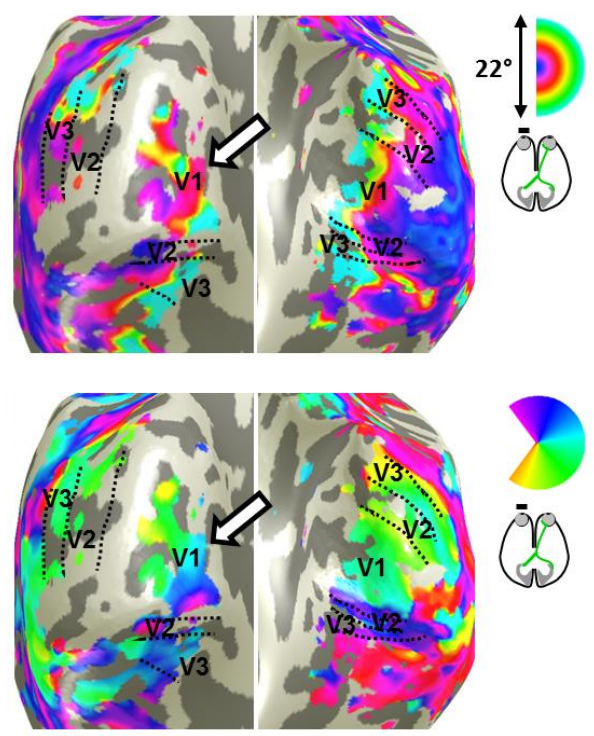
eye stimulation. Eccentricity (top row in each panel) and polar angle (bottom row in each panel) maps are depicted on the inflated occipital cortex of CHP. For left eye stimulation, orderly eccentricity and polar angle maps are obtained on left hemisphere for both right and left hemifield stimulation (A, B) and vice versa for right eye stimulation (C, D). In addition, there is normal input to the hemisphere contralateral to the stimulated eye (white arrows). It is small for 
left eye stimulation and sizable for right eye stimulation, where it spans the entire polar angle range.
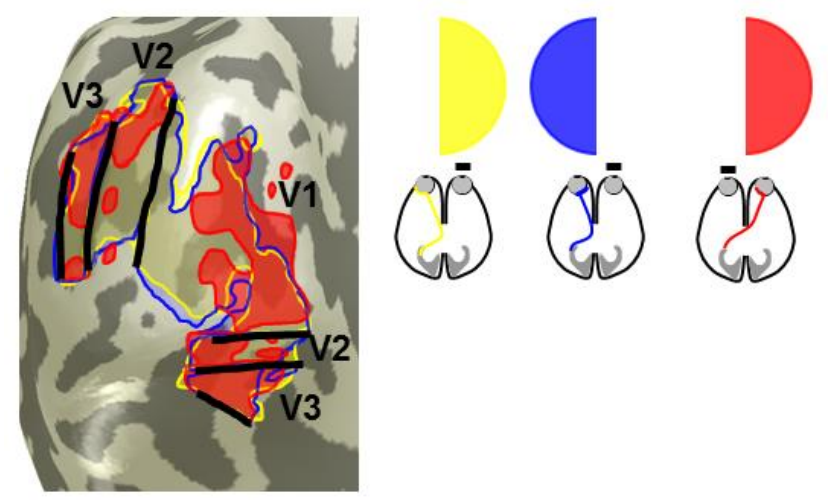

A)

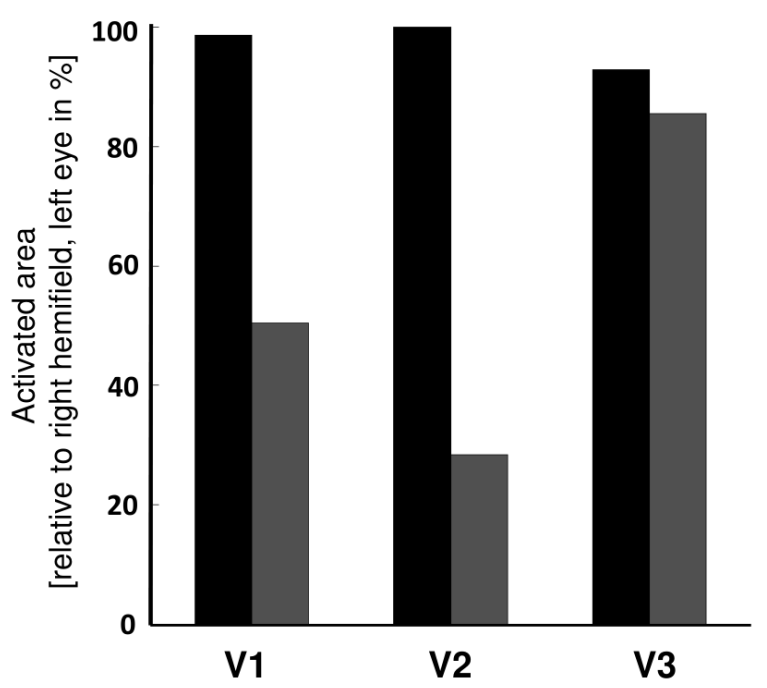

B)

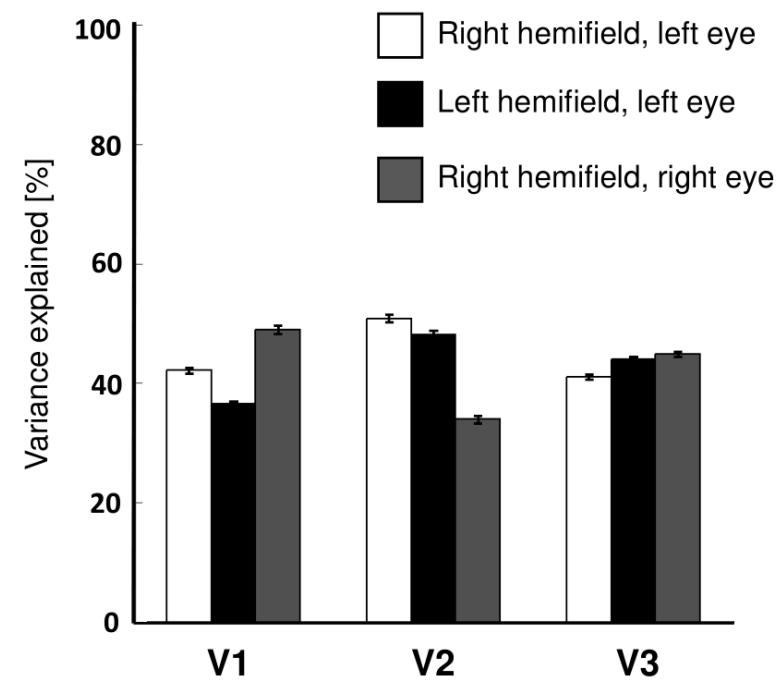

Figure 5. Activated area and goodness of pRF model fit across left hemisphere V1-V3. A) Activated area (normalized with respect to right hemifield, left eye stimulation condition) of left V1-V3 for left hemifield stimulation of the left eye (black bars) and right hemifield stimulation of the right eye (gray bars). For left hemifield stimulation of the left eye, the activated area of the left V1-V3 does not decrease below $92 \%$. For the right hemifield stimulation of the right eye, the relative activated area of $\mathrm{V} 1, \mathrm{~V} 2$ and, $\mathrm{V} 3$ is smaller, covering 50\%, 28\%, and $85 \%$, respectively. B) Comparison of the goodness of fit, i.e. mean variance explained (VE) $\pm \mathrm{SEM}$, of the pRF 
model between right and left hemifield stimulation of the left eye (white and black bars) and right

215 hemifield stimulation of the right eye (gray bars) in V1-V3 restricted to the overlapping area of

216 the three maps $\left(\mathrm{ROI}_{3 \mathrm{maps}}\right)$. The $\mathrm{VE}$ for all three maps is relatively similar in $\mathrm{V} 1$ and $\mathrm{V} 3$ ranging 217 from (49-37\%) and (41-45\%), respectively. For V2 it is reduced to $34 \%$ for the right hemifield 218 right eye condition.

Distinct neuronal populations with preference to left or right eye revealed by laminar analysis

To assess the fine-grain structure of the left V1 in CHP, which receives triple input from both hemifields, we revisited the submillimeter fMRI data. The differential responses to left and right eye stimulation were visualized on an anatomical image and across the cortical surface at multiple sampling depths (see Methods). Alternating and elongated patches were observed in an anterior ROI, ( $\mathrm{ROI}_{\text {signal }}$ ), drawn in the banks of the calcarine sulcus, demonstrating a differential preference for the left or the right eye (Figure 6A). The width of these patches was between 1 to 5 $\mathrm{mm}$. This variation is expected due to the effects of fMRI, namely, BOLD blur on cortical sampling and subsequent aliasing. To test the reproducibility, the data were split into two halves i.e. odd and even scans and the analysis was repeated for each half. Similar clustered patterns were observed for both halves, demonstrating scan-to-scan consistency (Figure S4). It should be noted that based on the current data, we cannot infer the spatial segregation of the ocular dominance domains even though the finer patches, specifically observed in the deep layer (Figures 6A and S4), provide an indication of these domains.

Furthermore, to quantitatively assess the presence of neural populations with ocular preference, a selectivity index ( $\mathrm{I}_{\text {selectivity }}$ ) was derived from the data, according to the approach used in previous studies (Kemper et al., 2018; Olman et al., 2016). It was defined as the difference between the responses to left and right eye stimulation divided by the sum of the responses to the visual stimuli (see Methods). In addition to the signal ROI, ten ROIs (ROIs noise) were drawn at different regions of occipito-temporal cortex, where no ocular dominance domains are expected (see Figure 6B, left panel). The selectivity index was compared between $\mathrm{ROI}_{\text {signal }}$ and each $\mathrm{ROI}_{\text {noise }}$ using receiver operating characteristic (ROC) analysis across superficial, middle and deep layers. An additional comparison was also performed between the selectivity in $\mathrm{ROI}_{\text {signal }}$ and the averaged selectivity of all the ten $\mathrm{ROI}_{\text {noise }}$. As illustrated in Figure $6 \mathrm{~B}$, the average selectivity 
0.5527, AUC for middle layer $=0.5689$, and AUC for superficial layer $=0.5835$ ). This suggests the segregation of two neuronal populations with preference to the left or the right eye, predominantly in the vicinity of calcarine sulcus. A similar segregation is expected at the level of the lateral geniculate nucleus (LGN), although no systematic activation was observed in this subcortical region. Due to the unavailability of CHP for further scanning, no data are available contrasting left and right hemifield representations to test for hemifield dominance domains as described before in complete achiasma (Olman et al., 2016).

A) Left eye vs right eye
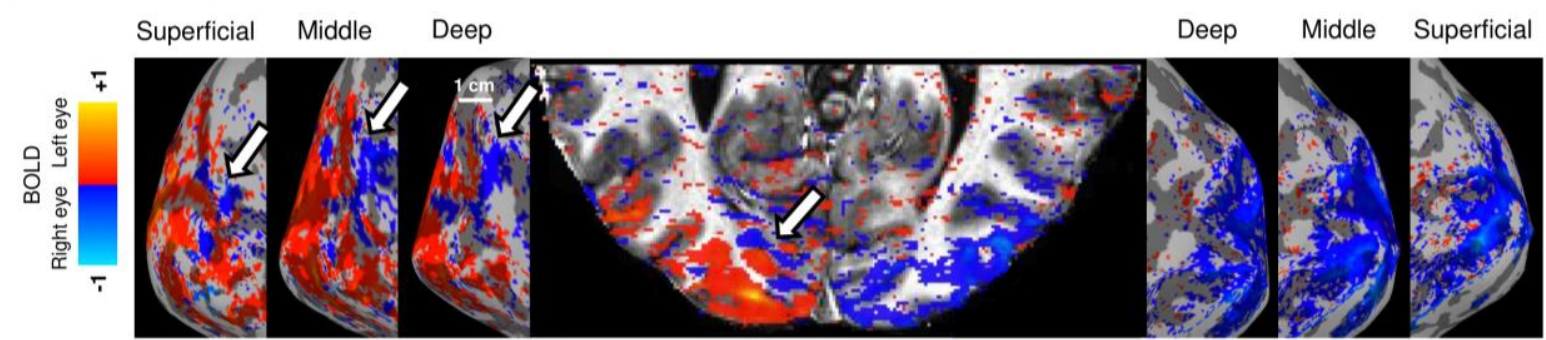

B)
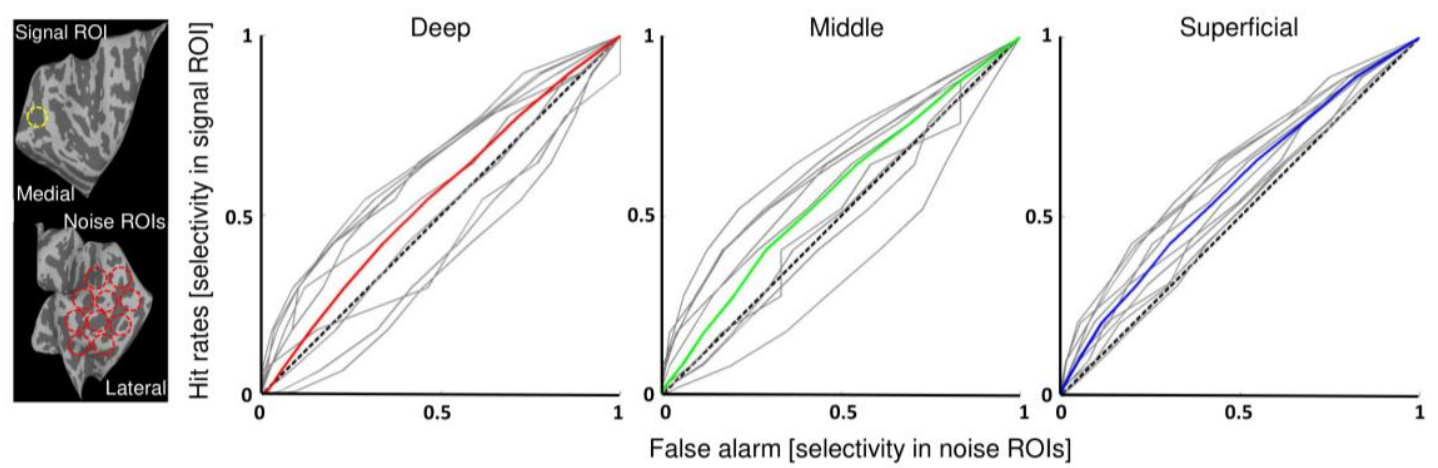

Figure 6. Response to left vs right eye stimulation. A) The cortical activation (signal amplitude expressed as $\beta$ coefficient from the general linear model thresholded by cluster size and mean Student's T statistic (cluster $=20$, threshold by $\mathrm{T}=1.98, \mathrm{p}=0.05$, uncorrected) is projected onto a clipped anatomical image of the occipital cortex and onto the inflated cortical surfaces of the deep, middle, and superficial layers. Right eye preference observed in the anterior region of the left calcarine sulcus is indicated by white arrows. B) Comparison of $\mathrm{I}_{\text {selectivity }}$ between signal and noise ROIs across lamina via ROC analysis. The approximate position of these ROIs is displayed in the left panel. Hit rates depict the selectivity in the signal ROI, and false alarms outline the selectivity in the ten noise ROIs. The dashed line represents bisection, where the selectivity indices of the signal and noise ROIs cannot be distinguished. The average selectivity indices for all three cortical layers are above the reference line, indicating segregation of two neuronal populations with differential responses to the left or the right eye. 


\section{Discussion}

270 In the case of chiasma hypoplasia examined here, input from three visual hemifields converges onto the same cortical area. This puts a critical challenge on the organization of the visual cortex,

272 which normally comprises a retinotopically aligned overlay of only two maps, i.e. input from 273 each eye representing the contralateral visual hemifield. The current study, therefore, provides 274 novel insight into the scope and mechanisms of human visual system development and plasticity. 275 Using submillimeter fMRI at 7T, DWI and fMRI-based pRF mapping at 3T, we report 276 asymmetrical crossing of the nasal fibers of the two eyes that results in three overlaid representations of opposing hemifields on the left visual cortex with segregation of two neuronal populations in the vicinity of the calcarine sulcus with different ocular preference. These findings demonstrate that the scope of cortical plasticity in the human visual system is sufficient to accommodate input from three visual hemifields.

The retinotopically registered overlay of the representation of visual hemifields is a key property of the primary visual cortex. Remarkably, this is not only observed in the neuro-typical visual system, where these two maps comprise the binocular input of the contralateral visual hemifield it also holds for conditions with abnormal predominantly monocular input, as achiasma, albinism, or FHONDA (Ahmadi et al., 2018; Hoffmann et al., 2012, 2003). While the two maps segregate into ocular dominance domains in the neuro-typical case, they segregate into hemifield domains (Guillery et al., 1984; Olman et al., 2016) for conditions with congenital chiasma malformations. This is taken as evidence for largely unaltered geniculo-striate connections despite congenitally abnormal input to the LGN (Hoffmann and Dumoulin, 2015), as summarized in Figure 7 A and B. In fact, it appears that the neuro-typical geniculo-striate projection is in general largely unaffected by enhanced or absent crossing at the optic chiasm as in albinism/FHONDA or achiasma, respectively (Ahmadi et al., 2018; Hoffmann and Dumoulin, 2015). Consequently, we asked which cortical organization pattern would result from such stability in the geniculo-cortical projections in the present case of chiasma hypoplasia, in whom the left V1 receives triple hemifield input. Such an input is expected to result in a combination of the normal organization, i.e. ocular dominance domains (Figure 7A), and the organization found in complete achiasma, i.e. hemifield domains (Figure 7B) as depicted in Figure 7C: the abnormal ipsilateral input from the left nasal hemiretina and the residual normal input from the right nasal hemiretina are expected to converge into the same domain (Figure 7C). In the absence of geniculo-striate rewiring, the 
301 hemifield, via the left eye, that is interleaved with combined retinotopic representations of the

302 ipsilateral and contralateral hemifield, via the left and right eye respectively. We, therefore, 303 termed it in analogy to the nomenclature introduced previously (Hoffmann and Dumoulin, 2015),

304 'Interleaved Combined Representation'. In fact, such a pattern would result in the macroscopic

305 cortical mapping we observed in the left occipital lobe. Moreover, it would predict, at the mesoscopic scale, regions that are more strongly activated by one eye than the other, and vice versa. This is in accordance with our submillimeter fMRI findings of two interdigitated neuronal populations with different eye preference on the left hemisphere. It should be noted though that the domain receiving input from the right visual hemifield, receives input from both eyes, thus reducing the differential activation via the two eyes. Further, we can, at present, not tell whether the neuronal populations representing the right hemifield input from both eyes segregate into distinct neuronal populations, due to the unavailability of data with sufficient resolution. Taken together, stable geniculo-striate projections still hold true even in the presence of triple input as observed in CHP. This conservative projection scheme, therefore, appears to be the most parsimonious concept to explain the cortical maps observed in a set of congenital projection abnormalities of the optic nerves, i.e. for enhanced, reduced or absent crossing.

317 Remarkably, the triple hemifield input to the left hemisphere affects only, albeit extensively, part 318 of the primary visual cortex. In fact, another part of the visual cortex receives largely exclusive 319 input from both hemiretinae of the left eye, as typical for complete achiasma. As a consequence, there is a coexistence of the 'Interleaved Representation' (Figure 7B) and 'Interleaved Combined Representation' (Figure 7C), occupying different regions of the left primary visual cortex. This is in accordance with the reports of animal models of albinism indicating a mixed organization patterns in the primary visual cortex (Cooper and Blasdel, 1980). Taken together, this suggests that the relevant adaptive developmental mechanisms can act locally.

325 Consistent with the reports on complete achiasma (Davies-Thompson et al., 2013; Hoffmann et al., 2012; Olman et al., 2016; Victor et al., 2000), the participant of the present study did not have visual field defects and made effective use of vision in daily life, including sport activities and reading. This indicates that in the current case of chiasma hypoplasia general aspects of visual function are preserved, apart from reduced binocular/stereo-vision, strabismus, and nystagmus. Despite the binocular input to the left visual cortex, the disruption of binocular and stereo-vision is expected in CHP due to vertical and horizontal deviations between the two eyes. This indicates 
333 although the independence of perception in opposite hemifields was not quantitatively tested

334 here, the every-day behavior of CHP does not show any confusion between the left and right

335 hemifields. As a consequence, it is, in analogy to findings in other conditions with chiasma

336 abnormalities (Klemen et al., 2012; Olman et al., 2016; Victor et al., 2000), suggested that the

337 three representations of the hemifields in the left primary visual cortex independently drive visual

338 perception. Further research addressing the independence of the three different maps is motivated

339 by the current findings. Based on our current knowledge, no integration of information across the

340 ocular dominance and/or across the hemifield dominance domains is expected to occur in CHP.

341 Thus, the plasticity of the intracortical micro-circuitry appears instrumental to cope with the

342 abnormal visual input and to support independent processing of the three superimposed

343 hemifields (Figure 7).

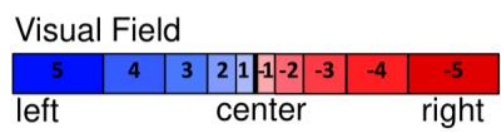

A) Control

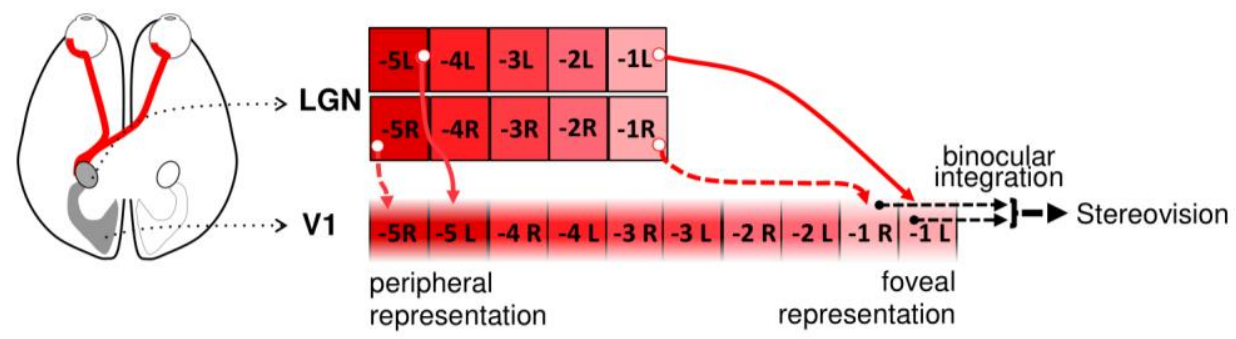

B) Complete achiasma

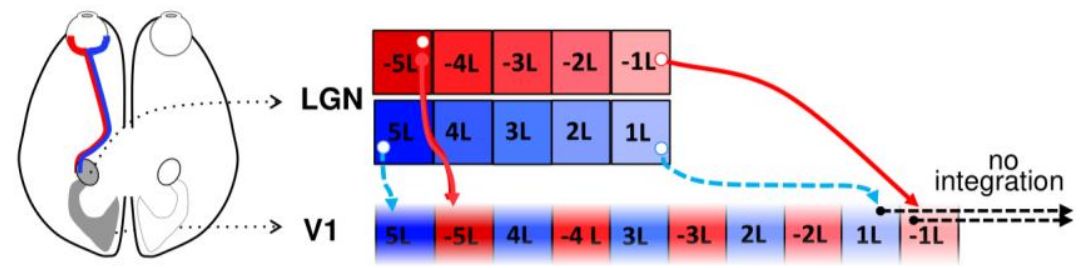

Interleaved representation

C) Chiasma hypoplasia

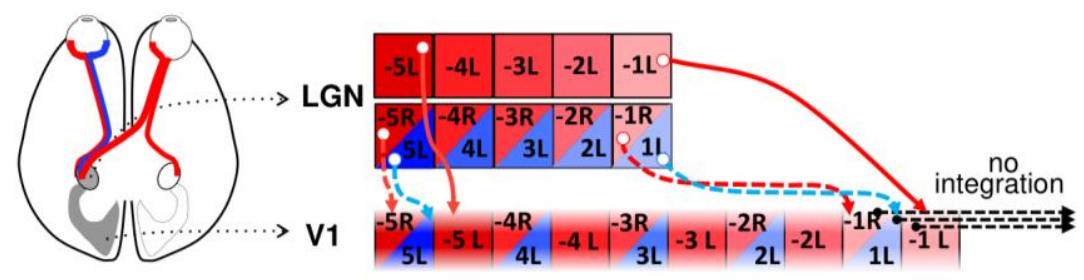

Interleaved combined representation 
Figure 7. Schematic of visual field representations in primary visual cortex for control, complete achiasma and chiasma hypoplasia. A) Control. The binocular input to the left LGN is organized as retinotopic maps of the right visual field (color coded red; negative numbers) that are separate for each eye (subscript indicates L - left, R - right eye input; the LGN is schematized as only two LGN layers with input from either eye). The geniculo-striate projections (solid red arrows for the left and dashed red arrows for the right eye input) result in interleaved retinotopic representations of the two eyes in V1. The integration of binocular input from corresponding locations in the contralateral visual field leads to binocular and stereo-vision. B) Complete achiasma. The left LGN receives monocular input from the nasal (blue) and from the temporal (red) hemiretina of the ipsilateral eye (i.e. left the eye, indicated by the subscript L). Consequently, there is in addition to the normal input from the contralateral visual field (red fields with negative numbers) input from the ipsilateral visual field (blue fields with positive numbers). This leads to an interleaved representation of opposing hemifields in V1, which is associated with a conservative, i.e. unchanged, geniculo-striate projection despite the abnormal LGN input (dashed cyan arrows). The absence of integration of the monocular input from opposing visual hemifields counteracts cross-talk of information between the hemifield. C) Chiasma hypoplasia. The left LGN receives binocular input from the contralateral visual field (red fields with negative numbers) as well as ipsilateral input (blue fields with positive numbers) only from the left eye. The triple hemifield input to the left LGN is organized as an interleaved representation of the contralateral visual field from the left eye (red fields with negative numbers in separate boxes) and combined representation of opposing hemifields from both eyes (red fields with negative numbers and blue fields with positive numbers in shared boxes). A conservative geniculo-striate projection to V1 would result in an interleaved combined representation pattern, obtained by the combination of cortical organization schemes for the control (A) and complete achiasma (B). Specifically, while the contralateral input of the left eye is incorporated via a separate domain, the contralateral input of the right eye together with the ipsilateral input of the left eye are assumed to be accommodated within a shared domain. Similar to complete achiasma, no integration is expected to occur across the three hemifield representations, supporting independent processing of the three maps.

Akin to other visual pathway abnormalities, it is therefore assumed that the aberrant representation in CHP is made available for relatively normal visual perception through the interplay of subcortical stability and cortical plasticity. The cortical plasticity might not be confined to changes in the intra-cortical connectivity and, in addition, affect the cortico-cortical connectivity as suggested by changes in $\mathrm{pRF}$ and connective field (CF) size estimates (Figure S3). It, therefore, appears that the extra-input from the right eye impacts on the cortico-cortical connectivity of the early visual areas in CHP. 
underlying developmental principles. A common limitation, however, is the rarity of relevant conditions and hence the limited availability of affected individuals. This also applies to the field of congenital malformations of the optic chiasm. While the well-known enhanced crossing of the optic nerves in albinism is already a rare condition [1.17:000; (Grønskov et al., 2007)], reduced crossing, i.e. achiasma, is much rarer [ $<50$ cases published worldwide, (Hoffmann and Dumoulin, 2015)]. In fact, fMRI-data have been reported in the past two decades for only 6 different individuals (Bao et al., 2015; Davies-Thompson et al., 2013; Hoffmann et al., 2012; Nguyen et al., 2018; Victor et al., 2000). Thus, investigating a subtype of achiasma, i.e. with the specific hypoplasia of the optic chiasm reported in the present study, is an exceptional case. As a consequence, we did not have the opportunity to obtain additional data for this condition, neither from the present nor from other individuals, despite the potentially informative value of e.g. additional submillimeter fMRI data. Another limitation of investigating visual system pathologies is related to fixation instabilities. These might alter the effective visual stimulation and thus impact on the obtained cortical activation patterns. For the individual of the present study, nystagmus was observed to be moderate, although no quantitative data are available. It should be noted, however, that while the present case is unique, it shares features, previously reported for achiasma, i.e. the retinotopic overlay of opposing visual hemifields (Hoffmann and Dumoulin, 2015). This is taken as an indication of the overall quality of the functional data obtained. Specifically, the data-set allowed reproducing previous results, i.e. orderly eccentricity and polarangle maps from opposing visual hemifields via the ipsilateral (left) eye, and adding a further feature, i.e. the third input to the left visual cortex via the contralateral (right) eye. Stimulusinduced deviations from central fixation would be expected to be specific to the visual stimuli applied. Importantly, the activation in the cortical region comprising the additional third input was reproducible for different stimulation conditions applied via the right eye, i.e., for bilateral stimulation (Figure 1B) and for right hemifield mapping (Figure 3D). We conclude that fixation instabilities are a highly unlikely source of the observed cortical triple maps. Finally, it might be argued that the comparison of the observed findings in CHP with strabismic amblyopes with a similar level of acuity would be more informative than the healthy controls. However, no 412 alteration/shrinkage of the ocular dominance domains has been reported in a previous 413 postmortem study of an individual with strabismic amblyopia (Horton and Hocking, 1996).

414 Furthermore, the retinotopic organization of the visual cortex in these patients does not drastically differ from the controls except for enlarged pRF sizes for the amblyopic eye 
416 (Clavagnier et al., 2015). It is, therefore, concluded that the interpretation of the observed striking

417 cortical organization in CHP does not depend on the reference group.

418 Conclusion

419 Congenital visual pathway abnormalities are powerful models to further our understanding of the 420 scope of developmental stability and plasticity in the human visual system, which may impact on 421 novel therapeutic approaches. Here, we demonstrate that the gross topography of the geniculo422 striate projections in CHP remains chiefly unaltered resulting in triple hemifield input to the 423 visual cortex. This reflects an unaltered geniculo-cortical axonal guidance by chemoaffinity 424 gradients (Cang et al., 2005; McLaughlin and O'Leary, 2005), even in the face of severely 425 erroneous input to LGN. The additional input to the left visual cortex is assumed to be 426 incorporated by sharing the same domain between the abnormal input of the left eye and normal 427 input of the right eye. This underlines that intra-cortical plasticity provides sufficient scope to 428 accommodate highly atypical visual input for comparatively normal visual processing.

\section{Materials and Methods}

\section{Participants}

431 The measurements were performed at two sites. CHP was first scanned at Magdeburg University, 432 Germany, at the age of 24. In two consecutive days, she underwent submillimeter fMRI at 7T and 433 DWI scanning sessions at 3T. Due to limited availability of CHP, pRF mapping data were 434 acquired two years later at York Neuroimaging Center, UK, at 3T. In addition to CHP, 12 435 respective control participants were also included in the current study. The first four controls $(\mathrm{C} 1$ 436 - C4) took part in a pRF mapping session at 3T while the other seven controls (C5 - C11) 437 participated in the DWI sessions. The last control participant (C12) underwent both 438 submillimeter fMRI and DWI at 7 and 3T. All the experiments on controls were conducted in 439 Magdeburg. Informed written consent was obtained from all participants prior to the study 440 investigations. The procedures followed the tenets of the declaration of Helsinki and the

441 respective protocols were approved by the ethical committees of the University of Magdeburg 442 and York Neuroimaging Centre.

443 Submillimeter fMRI

444 Visual stimulation: Visual stimuli were presented by back-projection onto a screen with a 445 resolution of $1920 \times 1080$ pixels and viewed at a distance of $100 \mathrm{~cm}$ via an angled mirror. 
446 Presentation software package (Neurobehavioral Systems, Berkeley, CA, USA) was used to 447 control stimulus presentation. The stimuli extended $\pm 12.9^{\circ}$ by $\pm 7.4^{\circ}$ of visual angle from the 448 center of the screen and comprised bilateral, contrast reversing ( 8 reversals per second) black and 449 white checkerboards with 24 segments and 26 rings (mean luminance $62 \mathrm{~cd} / \mathrm{m}^{2}$, contrast $99 \%$ ). A 450 block design, alternating between the two eyes was selected. It consisted of 14 checkerboard 451 presentation blocks ( 7 blocks per eye), each of which lasted for $12 \mathrm{~s}$ and was followed by a rest 452 block (mean luminance gray background) with the same duration. The presentation blocks were 453 preceded by an additional rest block of $12 \mathrm{~s}$ for dummy stimulation. Participants wore a custommade manually operated shutter that allowed monocular viewing through either the left or right eye. They fixated a central fixation cross, which changed its color one second after initiation of each rest block, lasting for $23 \mathrm{~s}$ (11 s of the rest block plus $12 \mathrm{~s}$ of the next presentation block).

457 The participants were requested to occlude the right eye and view the stimuli with the left eye for 458 a green fixation cross, and vice versa for a red one. An MRI-compatible camera was used to view the dominant eye, to ensure that the participants were doing the task correctly.

MRI acquisition: For functional imaging, T2*-weighted images were acquired using a 2D gradient-echo EPI sequence with a 7 Tesla whole body MRI scanner (Siemens Healthineers, Erlangen, Germany) using a 32 channel head coil (Nova Medical, Wilmington, MA). The acquisition parameters were as following: TR $|\mathrm{TE}=3000| 22 \mathrm{~ms}$, flip angle $=90^{\circ}, \mathrm{FOV}=169$ (right-left) $\times 130$ (anterior-posterior) $\times 27$ (feet-head) $\mathrm{mm}^{3}$, acceleration factor $(\mathrm{r})=4$ with GRAPPA reconstruction, phase-encoding direction $=$ right-left, phase partial Fourier $=5 / 8$, bandwidth $(\mathrm{BW})=1086 \mathrm{~Hz} / \mathrm{px}$, echo-spacing $=1.13 \mathrm{~ms}$ and voxel size $=0.65 \mathrm{x} 0.65 \mathrm{x} 0.65 \mathrm{~mm}^{3}$. Forty-one oblique axial slices were acquired parallel to the calcarine sulcus for the duration of 348 s with 116 time frames, of which the first four were discarded. Foam padding was used to minimize head motion. Four runs of bilateral stimulation were performed for each participant in a single session.

High-resolution anatomical volume was obtained using a 3D T1-weighted MPRAGE sequence $472\left(\mathrm{TR}|\mathrm{TE}| \mathrm{TI}=2500|2.76| 1050 \mathrm{~ms}\right.$, total duration $=14: 14 \mathrm{~min}$, flip angle=5 ${ }^{\circ}$, FOV: $350 \times 263 \times 350 \mathrm{~mm}^{3}$, and voxel size $\left.=0.65 \times 0.65 \times 0.65 \mathrm{~mm}^{3}\right)$. In addition, a proton density weighted volume without the inversion module (identical parameters except for TR $=1820 \mathrm{~ms}$

475 and total duration $=5: 33$ min) was acquired to correct for receive coil biases (Van de Moortele et 476 al., 2009). 
477 Data analysis: To obtain an inhomogeneity corrected anatomical volume, the T1-weighted

478 MPRAGE reference volume was divided by the proton density weighted volume. Gray and white

479 matter (GM/WM) were segmented based on the resulting anatomical volume in MIPAV

480 (https://mipav.cit.nih.gov/) using the TOADS/CRUISE algorithm (Bazin and Pham, 2007; Han et al., 2004). Manual editing was performed in ITK-GRAY

482 (https://web.stanford.edu/group/vista/cgi-bin/wiki/index.php/ItkGray) to minimize the

483 segmentation error. An equi-volume distance map was employed (Waehnert et al., 2014) to build

484 a coordinate system along the cortical depth, taking the local curvature into account.

485 The functional data were corrected for motion artifacts and spatial distortion using MCFLIRT 486 function of FSL (https://www.fmrib.ox.ac.uk/fsl) and a point spread function (PSF) mapping 487 method (In and Speck, 2012) respectively. Motion and distortion corrected data were then 488 analyzed using AFNI (https://afni.nimh.nih.gov/afni). Time series were averaged across 489 repetitions for each participant to increase the signal-to-noise ratio (SNR). Afterwards, the 490 averaged functional volume was aligned to the T1-weighted anatomical volume using an affine 491 transformation. The alignment was performed in three steps: First, the T1-weighted anatomy and 492 the averaged EPI were clipped in the anterior-posterior direction, leaving only the occipito493 temporal cortex. A good starting point was provided by centering the functional volume on the 494 anatomy using the respective centers of mass. Next, the averaged functional volume was affinely 495 aligned to the T1-weighted volume via AFNI's 'align_epi_anat.py' with the local Pearson's 496 coefficient (LPC) cost function (Saad et al., 2009), using the two-pass option. This procedure blurs the functional volume and initially allows for large rotation and shift, and then refines the alignment by an affine transformation. Finally, the resulting alignment was further improved via $3 \mathrm{~d}$ Allineate, using the one-pass option. In this step, the functional volume is not blurred. Only a small amount of shift and rotation is allowed, using an affine transformation that is obtained by concatenating the transformation matrices generated in previous steps (Fracasso et al., 2018; Klein et al., 2018).

503 A general linear model was used to analyze the functional data. For each voxel, the percentage of BOLD signal changes to stimulation of the left and right eye was estimated via 3 dDeconvolve function of AFNI. Nuisance regressors were modeled using polynomials up to the second order to remove any linear and quadratic trends. The GLM analysis was performed on the native EPI space. The obtained GLM maps (T-maps and beta-coefficient-maps; $\mathrm{T}=1.98, \mathrm{p}=0.05$, 
509 the affine transformation matrix estimated in the alignment step. For each of the cortical layers, a

$5103 \mathrm{D}$ mesh was generated using AFNI's IsoSurface function. To assess the presence of ocular 511 dominance domains structures in the data throughout the cortical depth, eleven ROIs were 512 selected on the cortical surface of the deep, middle and superficial layers and were mapped back 513 onto the volume dataset via $3 \mathrm{~d} S u r f 2 \mathrm{Vol}$ function for further analysis. The first ROI (ROI $\left.{ }_{\text {signal }}\right)$ 514 was drawn in the banks of the calcarine sulcus where the ocular dominance domains should be 515 located. The remaining ten ROIs $\left(\mathrm{ROIs}_{n o i s e}\right)$ were drawn in different regions of the occipito516 temporal cortex where there should be no ocular dominance domains (see Figure 6B, left panel). 517 The selectivity index was then derived (Kemper et al., 2018; Olman et al., 2016) from the voxels 518 within these ROIs. It was defined as a measure for eye preference, i.e. the difference between the 519 responses to left ('L') and right ('R') eye stimulation divided by sum of the responses to visual 520 stimuli: $\mathrm{I}_{\text {selectivity }}=|(\mathrm{L}-\mathrm{R}) /(\mathrm{L}+\mathrm{R})|$. The segregation of the binocular input was quantitatively 521 evaluated by voxelwise comparison of the selectivity between $\mathrm{ROI}_{\text {signal }}$, and each $\mathrm{ROI}_{\text {noise, }}$ using 522 receiver operating characteristic (ROC) analysis. Furthermore, the selectivity of ROI ${ }_{\text {signal }}$ was

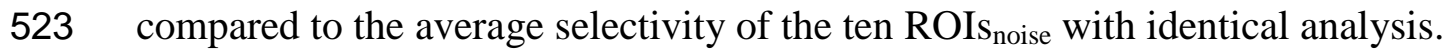

524 Diffusion-weighted imaging

525 MRI acquisition: DWI data were acquired using a 3 Tesla MAGNETOM Prisma syngo MR 526 D13D scanner (Siemens Healthineers, Erlangen, Germany) with a 64 channel head coil. MRI 527 acquisition was initiated by a localizer scan, followed by a T1-weighted and two diffusion528 weighted scans. All data were collected during a single scanning session. The T1-weighted volume was obtained in sagittal orientation using a 3D-MPRAGE sequence (TE | TR = 4.46 | $2600 \mathrm{~ms}, \mathrm{TI}=1100 \mathrm{~ms}$, flip angle $=7^{\circ}$, resolution $=0.9 \times 0.9 \times 0.9 \mathrm{~mm}^{3}, \mathrm{FoV}=230 \times 230 \mathrm{~mm}^{2}$, 531 image matrix $=256 \times 256 \times 176$, acquisition time $(\mathrm{TA})=11: 06 \mathrm{~min})$. The first diffusion532 weighted scan was acquired with Echo-Planar Imaging (EPI) with the following parameters: bvalue $=1600 \mathrm{~s} / \mathrm{mm}^{2}, \mathrm{TR}|\mathrm{TE}=9400| 64.0 \mathrm{~ms}$, voxel size $=1.5 \times 1.5 \times 1.5 \mathrm{~mm}^{3}$, phase-encoding 534 direction $=$ anterior to posterior, $\mathrm{FoV}=220 \times 220 \mathrm{~mm}^{2}$, and $\mathrm{TA}=22: 24 \mathrm{~min}$. Scanning was 535 performed with 128 unique gradient directions, thus the obtained diffusion-weighted data can be 536 described as High Angular Resolution Diffusion Imaging (HARDI) data (Tuch et al., 2002).

537 Gradient tables were generated using E. Caruyer's tool (http://www.emmanuelcaruyer.com/q538 space-sampling.php) for q-space sampling (Caruyer et al., 2013). Diffusion-weighted volumes 539 were evenly intersected by 10 non-diffusion weighted volumes for the purpose of motion correction. The second diffusion-weighted scan was acquired with identical parameters except for 
541 reversed phase-encoding direction in comparison to the preceding scan, i.e., posterior to anterior

542 direction. Acquisition of two diffusion-weighted scans with opposite phase-encoding directions

543 enhances the correction of susceptibility-induced geometric distortion (Andersson et al., 2003)

544 and improves the SNR of the total DWI data.

545 Data analysis: Conversion of DICOM images to NIFTI format, denoising of the DWI data and 546 removal of Gibbs ringing were performed with MRtrix 3.0 (http://www.mrtrix.org/). FSL was 547 employed for the correction of susceptibility-induced geometric distortions, eddy current 548 distortions, and motion artifacts. The bias field in the DWI data was corrected using ANTS 549 (http://stnava.github.io/ANTs/). Afterwards, DWI data were co-registered to the T1-weighted 550 volume, which was aligned beforehand to Anterior Commissure - Posterior Commissure line, via 551 mrDiffusion (https://github.com/vistalab/vistasoft/tree/master/mrDiffusion). The T1-weighted 552 volume was automatically segmented using FIRST function of FSL. Subsequently, manual 553 editing was performed to mitigate segmentation errors in the region of the optic chiasm.

554 Each voxel of the preprocessed DWI data was modelled using the Constrained Spherical 555 Deconvolution (CSD) approach (Tournier et al., 2008), which is particularly sensitive when 556 resolving populations of crossing fibers, like those observed in the optic chiasm, and benefits 557 from the high angular resolution of HARDI data. The application of the CSD model involved the 558 estimation of single fiber response function with Tournier's algorithm (Tournier et al., 2013) for 559 maximum harmonic order $(\operatorname{Lmax}=6)$ and the estimation of fiber orientation distribution 560 functions (Jeurissen et al., 2014) for 3 different maximum harmonic orders i.e. Lmax $=6,8$ and 561 10. Four ROIs were manually drawn on the T1-weighted volume, two covering cross-sections of 562 the two optic nerves, and the other two covering cross-sections of the two optic tracts. The ROIs 563 were placed as close to the optic chiasm as possible, but did not intersect it. Each ROI had a 564 width of 3 voxels (anterior-posterior) to assure proper streamline termination during 565 tractography. Fiber tracking was performed between the ROIs of the two optic nerves as seeds 566 and the ROIs of the two optic tracts as targets, resulting in 4 connectivity pairs ( 2 ipsilateral and 2 567 contralateral fiber bundles). Tracking was done in two directions i.e. from seed to target ROI and 568 backwards to ensure the indifference of the results to direction of tracking. The corresponding 569 generated connectivity pairs were subsequently merged together. The tracking employed an 570 ensemble tractography (ET) framework (Takemura et al., 2016), where tracking is performed 571 several times, each time for a different set of parameters. As such, the bias in the outcome tracts, 572 caused by parameter selection, is avoided. The tracking was performed with the probabilistic 
573 tracking algorithm iFOD2 (Tournier et al., 2010) using unique combinations of 2 different

574 fractional anisotropy $(\mathrm{FA})$ thresholds $(\mathrm{FA}=0.04$ and 0.08$), 3$ maximum curvature angles $\left(30^{\circ}\right.$,

$\left.57545^{\circ}, 60^{\circ}\right)$, and 3 CSD models estimated for different maximum harmonic orders $\left(\mathrm{L}_{\max }=6,8,10\right)$

576 for each of 139000 seeding attempts. Additionally, tractography employed an anatomically-

577 constrained tractography (ACT) approach (Smith et al., 2012), which constrains tractography

578 with anatomical priors derived from the anatomical image using white/gray matter, subcortical

579 gray matter and CSF masks obtained with FSL's FIRST function. As a result of the tractography,

5804 streamline groups corresponding to 4 distinct connectivity pairs were obtained. The proportion

581 of streamlines in each group was subsequently used as an estimate of the connectivity strength in

582 the optic chiasm.

583 Population receptive field ( $p R F)$ and connective field $(C F)$ modeling

584 Visual stimulation: Visual stimuli consisted of drifting bar apertures (stimulus size in York and 585 Magdeburg: $11^{\circ}$ and $10^{\circ}$ radius, respectively), exposing a moving high-contrast checkerboard 586 pattern (Dumoulin and Wandell, 2008) at four different directions i.e. upward, downward, left 587 and right. The bars were presented to each eye separately within a mask, covering either the left 588 or the right hemifields for stimulation of either the nasal or the temporal retina in separate 589 experiments. The width of the bars subtended one-quarter of the stimulus radius. Each pass of the 590 bars lasted for $30 \mathrm{~s}$, followed by a mean luminance block (zero contrast) of $30 \mathrm{~s}$. The stimuli were 591 generated in MATLAB (Mathworks, Natick, MA, USA) using the Psychtoolbox (Brainard, 1997; 592 Pelli, 1997) and rear-projected onto a screen (screen resolution in York and Magdeburg: 1920 x 5931080 and $1140 \times 780$ pixels, respectively) inside the magnet bore. In York, the participant (CHP) 594 viewed the screen at a distance of $57 \mathrm{~cm}$ via an angled, front-silvered mirror whereas the eye to 595 screen distance in Magdeburg was $35 \mathrm{~cm}$. Participants were required to fixate a centered dot and 596 to report color changes between red and green by means of a button press.

597 MRI acquisition: Identical 3 Tesla Prisma scanners (Siemens Healthineers, Erlangen, Germany) 598 were used at both sites. At York Neuroimaging Center, functional T2*-weighted images were 599 acquired with a 64 channel head coil. A total of 30 EPI slices were obtained within a FOV of 192 $600 \mathrm{~mm}$, with $3 \times 3 \times 3 \mathrm{~mm}^{3}$ voxels $\left(\mathrm{TR}|\mathrm{TE}=1500| 26 \mathrm{~ms}\right.$ and flip angle $\left.=80^{\circ}\right)$. Each functional 601 scan comprised 168 time frames, lasting for 252 s. The first eight time-frames (12 s) were 602 removed to allow magnetization to reach a steady-state. Foam padding was used to minimize 603 head motion. Additionally, a T1-weighted anatomical volume was acquired at a resolution of $1 \mathrm{x}$ $6041 \times 1 \mathrm{~mm}^{3}\left(\mathrm{TR}|\mathrm{TE}=2500| 42.26 \mathrm{~ms}\right.$ and flip angle $\left.=7^{\circ}\right)$. Eight functional scans were obtained 
605 in a single session (4 scans per eye). The right eye was stimulated during the first 4 runs while the

606 left eye was patched. The stimulation of each of the left and right hemifields was repeated twice 607 in a counterbalanced manner. After a short break in the scanning, the left eye was stimulated 608 while the right eye was occluded. The same stimulation procedure was performed for the left eye. 609 At Magdeburg University, functional images (TR $|\mathrm{TE}=1500| 30 \mathrm{~ms}$ and flip angle $=70^{\circ}$ ) were 610 acquired at a resolution of $2.5 \times 2.5 \times 2.5 \mathrm{~mm}^{3}$ with 54 axial slices, using a 64 channel head coil.

611 Every functional scan had 168 time frames (252 s). In addition, a high resolution whole-brain 612 anatomical volume (voxel size $=0.9 \times 0.9 \times 0.9 \mathrm{~mm}^{3}$, TR $|\mathrm{TE}=2600| 4.46 \mathrm{~ms}$, and flip angle $=$ $6137^{\circ}$ ) was obtained. Foam padding limited the head movements. In each session, left and right 614 hemifield stimulation conditions were performed monocularly and repeated six times (three 615 repetitions per hemifield).

616 Data analysis: The same analysis pipeline was used for data sets acquired in both sites. The T1617 weighted anatomical volume was automatically segmented using the recon-all function of 618 FreeSurfer (https://surfer.nmr.mgh.harvard.edu). The cortical surface was reconstructed at the 619 white/gray matter boundary and rendered as a smoothed 3D mesh (Wandell et al., 2000). The 620 MCFLIRT function of FSL was used for motion correction of the functional data. Motion 621 corrected data were then analyzed using freely available Vistasoft software package for 622 MATLAB (https://github.com/vistalab/vistasoft). Time series for the same conditions were 623 averaged together for each participant to increase the SNR. Afterwards, the averaged functional image was co-registered to the anatomical scan using a combination of Vistasoft and Kendrick 625 Kay's alignment tools (https://github.com/kendrickkay/alignvolumedata). Visual areas were 626 mapped using the population receptive field (pRF) modeling (Dumoulin and Wandell, 2008). 627 Briefly, the BOLD (blood oxygen level dependent) response of each voxel was predicted using a 628 2D-Gaussian model of the neuronal populations defined by three stimulus-referred parameters i.e. $629 \mathrm{x} 0, \mathrm{y} 0, \sigma$ where $\mathrm{x} 0$ and $\mathrm{y} 0$ are the coordinates of the receptive field center and $\sigma$ is it's spread 630 (Dumoulin and Wandell, 2008; Fracasso et al., 2016; Harvey and Dumoulin, 2011). The 631 predicted BOLD signal was then calculated by convolution of the stimulus sequence for the 632 respective pRF-model and its three parameters with the canonical hemodynamic response 633 function (Friston et al., 1998). The optimal pRF parameters were found by minimizing the sum of 634 squared errors (RSS) between the predicted and observed BOLD time-course. For all subsequent 635 analyses including derivation of the polar angle and eccentricity maps, required for the 
636 delineation of the visual areas, and the visualization on the inflated cortical surface, only the

637 voxels were included whose pRF fits exceeded $15 \%$ of the variance explained.

638 The connective field parameters were estimated from the fMRI time-series, using CF modeling 639 method that predicts the neuronal activity in one brain area with reference to aggregate activity in 640 another area (Haak et al., 2013). The BOLD response in each voxel of a target ROI i.e. V2 or V3, 641 was predicted with a symmetrical, circular 2D Gaussian CF model folded to follow the cortical 642 surface of the source ROI, i.e. V1. The CF model was defined by two parameters i.e. Gaussian 643 position and spread across the cortical surface. The optimal CF parameters were determined by 644 minimizing the residual sum of squares between the predicted, and the observed time-series. For 645 this purpose, many fMRI time-series predictions were generated by changing the CF positions 646 across all voxel positions and Gaussian spread values on the surface of the source ROI. Best 647 models were selected when the explained variance in the fMRI time-series survived a threshold 648 of $15 \%$.

\section{Visual field testing}

650 We simulated the Humphrey visual field testing using PsychoPy (https://www.psychopy.org) on 651 a calibrated CRT monitor (22-inch Mitsubishi 2070SB at $85 \mathrm{~Hz}$ ). Background luminance was set 652 to $10 \mathrm{~cd} / \mathrm{m}^{2}$, equal to $30 \mathrm{~dB}$. Goldmann size III stimuli i.e., white circular patches $\left(0.43^{\circ}\right.$ 653 diameter) were displayed for $235 \mathrm{~ms}$ and placed at 54 locations according to the Humphrey 24-2 654 standard test. In addition, four stimuli were placed at 12, 15, 18, and 21 degrees into the temporal 655 field along the horizontal meridian in order to capture the blind spot. The detection threshold was 656 tested in both eyes with one-up-one down staircase procedure with a minimum of 30 trials per 657 location. Responses were within $800 \mathrm{~ms}$ after stimulus presentation. An initial adaptive staircase 658 with $4 \mathrm{~dB} / 2 \mathrm{~dB}$ step sizes was used to coarsely estimate the threshold at 16 locations in the visual 659 field (4 in each visual quadrant), starting at the maximum gun value. Subsequently, a second 660 adaptive staircase with finer step sizes (minimum $0.25 \mathrm{~dB}$ ) was used to more accurately find the 661 threshold starting at a gun value of $25 \%$ of the maximum $\left(35 \mathrm{~cd} / \mathrm{m}^{2}\right)$.

\section{Acknowledgements}

663 The authors thank CHP and the controls for their patience and cooperation. This work was 664 supported by European Union's Horizon 2020 research and innovation programme under the 665 Marie Sklodowska-Curie grant agreement (No. 641805) to S.O.D, A.B.M and M.B.H. 


\section{Author Contributions}

668 Conceptualization, K.A., A.F., A.D.G., S.O.D., A.B.M., M.B.H.; Methodology, K.A., A.F., R.J.P

669 A.D.G., R.Y., O.S., J.K., F.P., M.B.H.; Formal Analysis, K.A., A.F., and R.J.P.; Investigation, 670 K.A., A.D.G., A.B.M., and M.B.H; Writing - Original Draft, K.A. and R.J.P; Writing - Review 671 and Editing, K.A., A.F., O.S., F.P., S.O.D., A.B.M., and M.B.H.

\section{Competing interests}

673 The authors declare no competing interests.

\section{References}

675

676

677

678

679

680

681

682

683

684

685

686

687

688

689

690

691

692

693

694

695

696

697

698

699

700

701

702

703

704

705

706

Ahmadi, K., Fracasso, A., van Dijk, J.A., Kruijt, C., van Genderen, M., Dumoulin, S.O., Hoffmann, M.B., 2018. Altered organization of the visual cortex in FHONDA syndrome. NeuroImage.

Andersson, J.L., Skare, S., Ashburner, J., 2003. How to correct susceptibility distortions in spin-echo echo-planar images: application to diffusion tensor imaging. Neuroimage 20, 870-888.

Apkarian, P., Bour, L., Barth, P.G., 1994. A unique achiasmatic anomaly detected in non-albinos with misrouted retinal-fugal projections. Eur. J. Neurosci. 6, 501-507.

Apkarian, P., Reits, D., Spekreijse, H., Van Dorp, D., 1983. A decisive electrophysiological test for human albinism. Electroencephalogr. Clin. Neurophysiol. 55, 513-531.

Bao, P., Purington, C.J., Tjan, B.S., 2015. Using an achiasmic human visual system to quantify the relationship between the fMRI BOLD signal and neural response. eLife 4, e09600. https://doi.org/10.7554/eLife.09600

Bazin, P.-L., Pham, D.L., 2007. Topology-preserving tissue classification of magnetic resonance brain images. IEEE Trans. Med. Imaging 26, 487-496.

Brainard, D.H., 1997. The Psychophysics Toolbox. Spat. Vis. 10, 433-436.

Cang, J., Kaneko, M., Yamada, J., Woods, G., Stryker, M.P., Feldheim, D.A., 2005. Ephrin-as guide the formation of functional maps in the visual cortex. Neuron 48, 577-589.

Caruyer, E., Lenglet, C., Sapiro, G., Deriche, R., 2013. Design of multishell sampling schemes with uniform coverage in diffusion MRI. Magn. Reson. Med. 69, 1534-1540.

Clavagnier, S., Dumoulin, S.O., Hess, R.F., 2015. Is the cortical deficit in amblyopia due to reduced cortical magnification, loss of neural resolution, or neural disorganization? J. Neurosci. 35, 14740-14755.

Cooper, M.L., Blasdel, G.G., 1980. Regional variation in the representation of the visual field in the visual cortex of the Siamese cat. J. Comp. Neurol. 193, 237-253.

Davies-Thompson, J., Scheel, M., Lanyon, L.J., Barton, J.J.S., 2013. Functional organisation of visual pathways in a patient with no optic chiasm. Neuropsychologia 51, 1260-1272. https://doi.org/10.1016/j.neuropsychologia.2013.03.014

Dumoulin, S.O., Wandell, B.A., 2008. Population receptive field estimates in human visual cortex. NeuroImage 39, 647-660. https://doi.org/10.1016/j.neuroimage.2007.09.034

Fracasso, A., Koenraads, Y., Porro, G.L., Dumoulin, S.O., 2016. Bilateral population receptive fields in congenital hemihydranencephaly. Ophthalmic Physiol. Opt. 36, 324-334.

Fracasso, A., Luijten, P.R., Dumoulin, S.O., Petridou, N., 2018. Laminar imaging of positive and negative BOLD in human visual cortex at 7 T. Neuroimage 164, 100-111. 
707

708

709

710

711

712

713

714

715

716

717

718

719

720

721

722

723

724

725

726

727

728

729

730

731

732

733

734

735

736

737

738

739

740

741

742

743

744

745

746

747

748

749

750

Friston, K.J., Fletcher, P., Josephs, O., Holmes, A., Rugg, M.D., Turner, R., 1998. Event-related fMRI: characterizing differential responses. NeuroImage $4, \quad 30$. https://doi.org/10.1006/nimg.1997.0306

Grønskov, K., Ek, J., Brondum-Nielsen, K., 2007. Oculocutaneous albinism. Orphanet J. Rare Dis. 2, 43.

Guillery, R.W., Hickey, T.L., Kaas, J.H., Felleman, D.J., Debruyn, E.J., Sparks, D.L., 1984. Abnormal central visual pathways in the brain of an albino green monkey (Cercopithecus aethiops). J. Comp. Neurol. 226, 165-183. https://doi.org/10.1002/cne.902260203

Haak, K.V., Winawer, J., Harvey, B.M., Renken, R., Dumoulin, S.O., Wandell, B.A., Cornelissen, F.W., 2013. Connective field modeling. Neuroimage 66, 376-384.

Han, X., Pham, D.L., Tosun, D., Rettmann, M.E., Xu, C., Prince, J.L., 2004. CRUISE: cortical reconstruction using implicit surface evolution. NeuroImage 23, 997-1012.

Harvey, B.M., Dumoulin, S.O., 2011. The relationship between cortical magnification factor and population receptive field size in human visual cortex: constancies in cortical architecture. J. Neurosci. Off. J. Soc. Neurosci. 31, 13604-13612. https://doi.org/10.1523/JNEUROSCI.257211.2011

Hoffmann, M.B., Dumoulin, S.O., 2015. Congenital visual pathway abnormalities: a window onto cortical stability and plasticity. Trends Neurosci. 38, 55-65. https://doi.org/10.1016/j.tins.2014.09.005

Hoffmann, M.B., Kaule, F.R., Levin, N., Masuda, Y., Kumar, A., Gottlob, I., Horiguchi, H., Dougherty, R.F., Stadler, J., Wolynski, B., Speck, O., Kanowski, M., Liao, Y.J., Wandell, B.A., Dumoulin, S.O., 2012. Plasticity and stability of the visual system in human achiasma. Neuron 75, 393-401. https://doi.org/10.1016/j.neuron.2012.05.026

Hoffmann, M.B., Seufert, P.S., Schmidtborn, L.C., 2007. Perceptual relevance of abnormal visual field representations: static visual field perimetry in human albinism. Br. J. Ophthalmol. 91, 509-513. https://doi.org/10.1136/bjo.2006.094854

Hoffmann, M.B., Tolhurst, D.J., Moore, A.T., Morland, A.B., 2003. Organization of the visual cortex in human albinism. J. Neurosci. Off. J. Soc. Neurosci. 23, 8921-8930.

Horton, J.C., Hocking, D.R., 1996. Pattern of ocular dominance columns in human striate cortex in strabismic amblyopia. Vis. Neurosci. 13, 787-795.

Huberman, A.D., Feller, M.B., Chapman, B., 2008. Mechanisms underlying development of visual maps and receptive fields. Annu Rev Neurosci 31, 479-509.

In, M.-H., Speck, O., 2012. Highly accelerated PSF-mapping for EPI distortion correction with improved fidelity. Magn. Reson. Mater. Phys. Biol. Med. 25, 183-192.

Jeurissen, B., Tournier, J.-D., Dhollander, T., Connelly, A., Sijbers, J., 2014. Multi-tissue constrained spherical deconvolution for improved analysis of multi-shell diffusion MRI data. NeuroImage 103, 411-426.

Kaule, F.R., Wolynski, B., Gottlob, I., Stadler, J., Speck, O., Kanowski, M., Meltendorf, S., BehrensBaumann, W., Hoffmann, M.B., 2014. Impact of chiasma opticum malformations on the organization of the human ventral visual cortex. Hum. Brain Mapp. 35, 5093-5105. https://doi.org/10.1002/hbm.22534

Kemper, V.G., De Martino, F., Emmerling, T.C., Yacoub, E., Goebel, R., 2018. High resolution data analysis strategies for mesoscale human functional MRI at 7 and $9.4 \mathrm{~T}$. NeuroImage 164, 48-58.

Klein, B.P., Fracasso, A., van Dijk, J.A., Paffen, C.L., Te Pas, S.F., Dumoulin, S.O., 2018. Cortical depth dependent population receptive field attraction by spatial attention in human V1. NeuroImage $176,301-312$. 
Klemen, J., Hoffmann, M.B., Chambers, C.D., 2012. Cortical plasticity in the face of congenitally altered input into V1. Cortex J. Devoted Study Nerv. Syst. Behav. 48, 1362-1365. https://doi.org/10.1016/j.cortex.2012.03.012

McLaughlin, T., O'Leary, D.D., 2005. Molecular gradients and development of retinotopic maps. Annu Rev Neurosci 28, 327-355.

Muckli, L., Naumer, M.J., Singer, W., 2009. Bilateral visual field maps in a patient with only one hemisphere. Proc. Natl. Acad. Sci. 106, 13034-13039. https://doi.org/10.1073/pnas.0809688106

Nguyen, C.T., Goh, C., Desmond, P., Abel, L.A., Lim, C.H., Symons, R.A., Hardy, T.G., 2018. Congenital achiasma and see-saw nystagmus in VATER syndrome association with hydrocephalus. J. Clin. Neurosci. 51, 63-65.

Olman, C.A., Bao, P., Engel, S.A., Grant, A.N., Purington, C., Qiu, C., Schallmo, M.-P., Tjan, B.S., 2016. Hemifield columns co-opt ocular dominance column structure in human achiasma. Neuroimage.

Pelli, D.G., 1997. The VideoToolbox software for visual psychophysics: transforming numbers into movies. Spat. Vis. 10, 437-442.

Saad, Z.S., Glen, D.R., Chen, G., Beauchamp, M.S., Desai, R., Cox, R.W., 2009. A new method for improving functional-to-structural MRI alignment using local Pearson correlation. Neuroimage 44, 839-848.

Smith, R.E., Tournier, J.-D., Calamante, F., Connelly, A., 2012. Anatomically-constrained tractography: improved diffusion MRI streamlines tractography through effective use of anatomical information. Neuroimage 62, 1924-1938.

Takemura, H., Caiafa, C.F., Wandell, B.A., Pestilli, F., 2016. Ensemble tractography. PLoS Comput. Biol. 12, e1004692.

Tootell, R.B., Mendola, J.D., Hadjikhani, N.K., Liu, A.K., Dale, A.M., 1998. The representation of the ipsilateral visual field in human cerebral cortex. Proc. Natl. Acad. Sci. 95, 818-824.

Tournier, J.-D., Calamante, F., Connelly, A., 2013. Determination of the appropriate b value and number of gradient directions for high-angular-resolution diffusion-weighted imaging. NMR Biomed. 26, $1775-1786$.

Tournier, J.D., Calamante, F., Connelly, A., 2010. Improved probabilistic streamlines tractography by 2nd order integration over fibre orientation distributions, in: Proceedings of the International Society for Magnetic Resonance in Medicine. p. 1670.

Tournier, J.-D., Yeh, C.-H., Calamante, F., Cho, K.-H., Connelly, A., Lin, C.-P., 2008. Resolving crossing fibres using constrained spherical deconvolution: validation using diffusion-weighted imaging phantom data. Neuroimage 42, 617-625.

Tuch, D.S., Reese, T.G., Wiegell, M.R., Makris, N., Belliveau, J.W., Wedeen, V.J., 2002. High angular resolution diffusion imaging reveals intravoxel white matter fiber heterogeneity. Magn. Reson. Med. Off. J. Int. Soc. Magn. Reson. Med. 48, 577-582.

Van de Moortele, P.-F., Auerbach, E.J., Olman, C., Yacoub, E., Uğurbil, K., Moeller, S., 2009. T1 weighted brain images at 7 Tesla unbiased for Proton Density, T2* contrast and RF coil receive B1 sensitivity with simultaneous vessel visualization. Neuroimage 46, 432-446.

Victor, J.D., Apkarian, P., Hirsch, J., Conte, M.M., Packard, M., Relkin, N.R., Kim, K.H., Shapley, R.M., 2000. Visual function and brain organization in non-decussating retinal-fugal fibre syndrome. Cereb. Cortex N. Y. N 1991 10, 2-22.

von dem Hagen, E.A.H., Hoffmann, M.B., Morland, A.B., 2008. Identifying human albinism: a comparison of VEP and fMRI. Invest. Ophthalmol. Vis. Sci. 49, 238-249. https://doi.org/10.1167/iovs.07-0458 
796

801

802

803

804

805
Waehnert, M.D., Dinse, J., Weiss, M., Streicher, M.N., Waehnert, P., Geyer, S., Turner, R., Bazin, P.-L., 2014. Anatomically motivated modeling of cortical laminae. Neuroimage 93, 210-220.

Wandell, B.A., Chial, S., Backus, B.T., 2000. Visualization and measurement of the cortical surface. J. Cogn. Neurosci. 12, 739-752.

Wandell, B.A., Dumoulin, S.O., Brewer, A.A., 2007. Visual field maps in human cortex. Neuron 56, 366383.

Weber, K.P., Landau, K., 2013. Teaching NeuroImages: Mind the gap! Postfixational blindness due to traumatic rupture of the optic chiasm. Neurology 80, e197-e198.

\section{Supplementary figures}

A) Left eye vs rest block

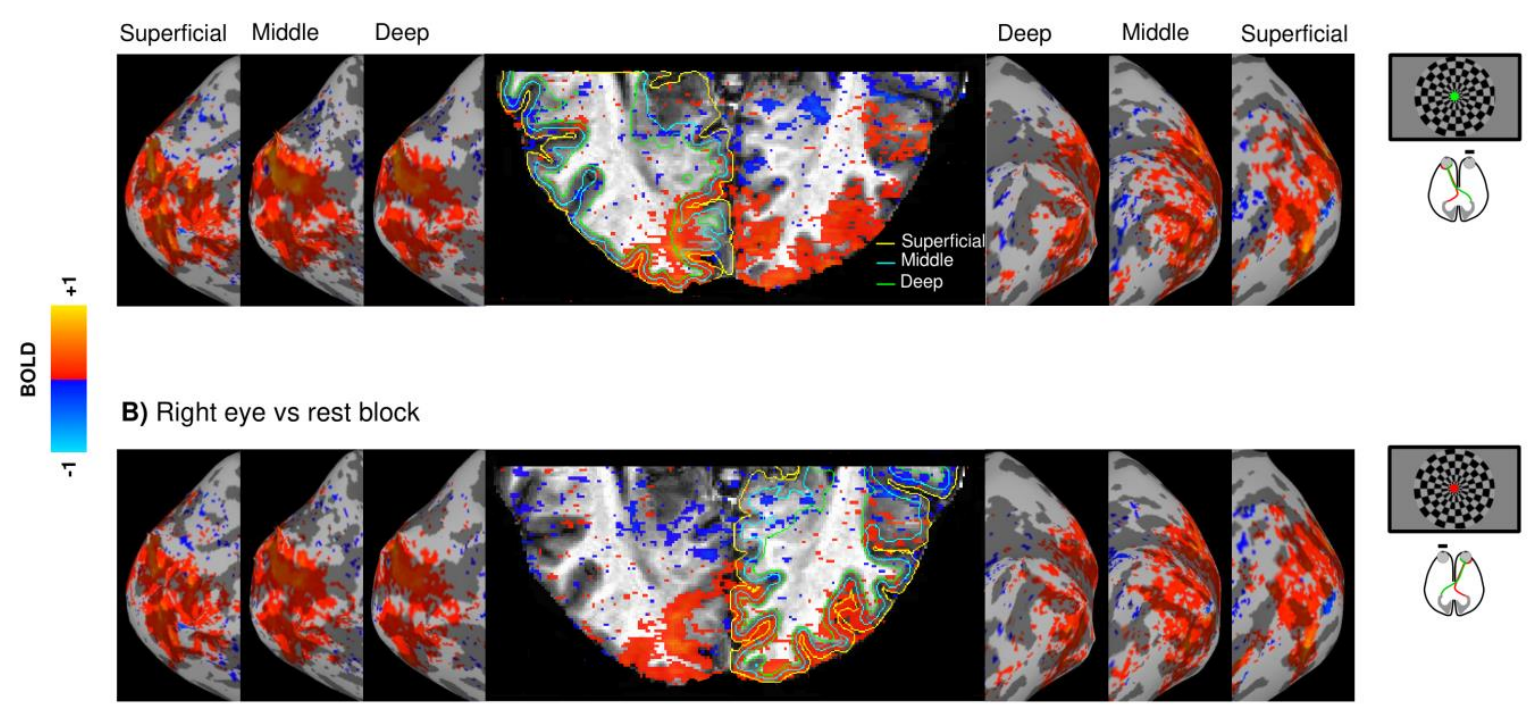

Figure S1: Cortical response lateralization during bilateral stimulation of each eye in a control participant. The cortical activation is projected onto a clipped anatomical image of the occipital cortex and onto the inflated cortical surfaces of the deep, middle, and superficial layers. Both left (A) and right (B) eye stimulation vs rest elicit bilateral activation. Conventions as for Figure 1. 
A) Left hemifield

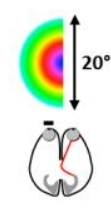

B) Right hemifield
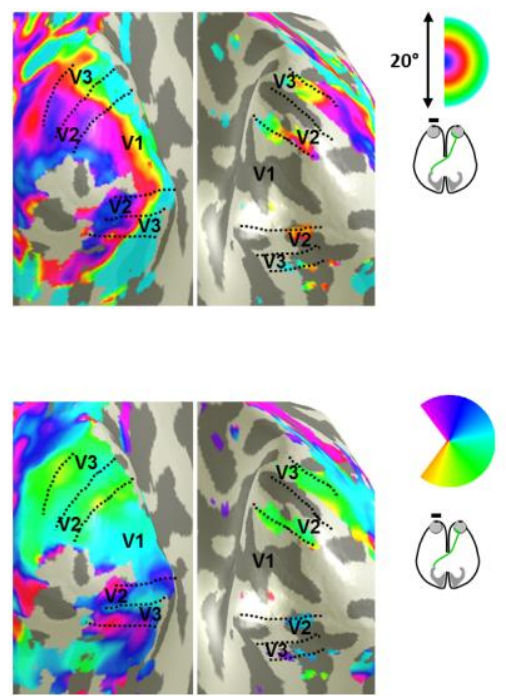
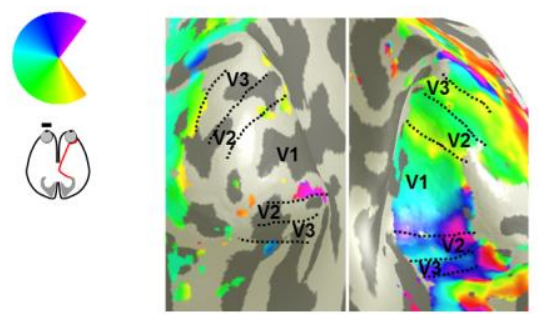

814

Figure S2: Visual field representations for unilateral stimulation of the right eye in a control participant. Eccentricity (top row) and polar angle maps (bottom row) on the inflated occipital cortex for left (A) and right (B) hemifield stimulation. In both cases, orderly eccentricity and polar angle maps were obtained predominantly on the hemisphere contralateral to the stimulated hemifield. Residual ipsilateral representations of the vertical meridians and fovea were observed in V1-V3 as reported previously (Hoffmann et al., 2003; Tootell et al., 1998). Note that this residual representation is clearly different from the additional third hemifield map in CHP (Figure 3D) which is more widespread and follows a retinotopic progression.

822
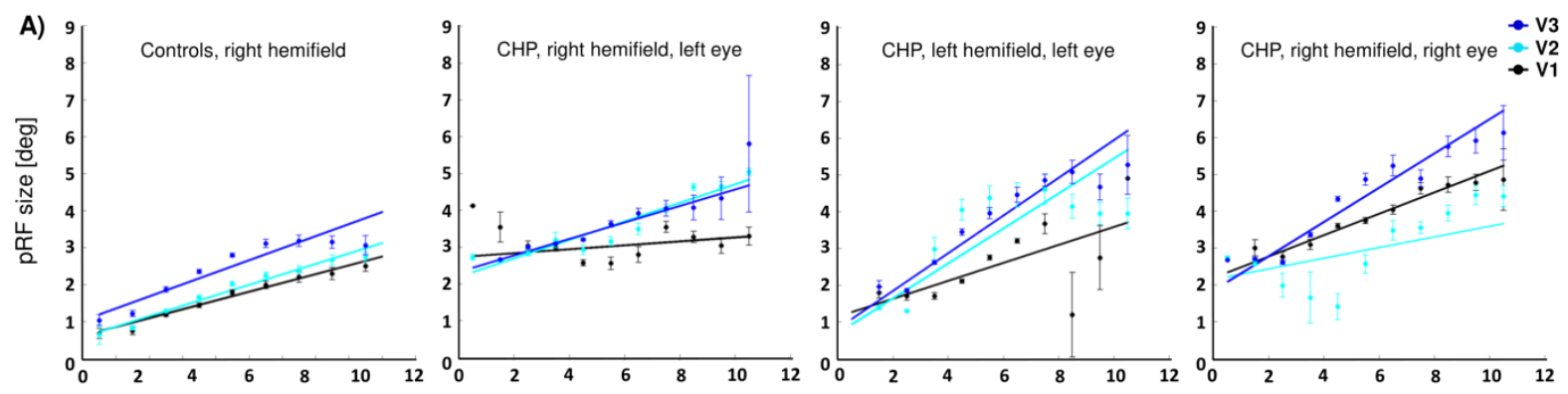

B)
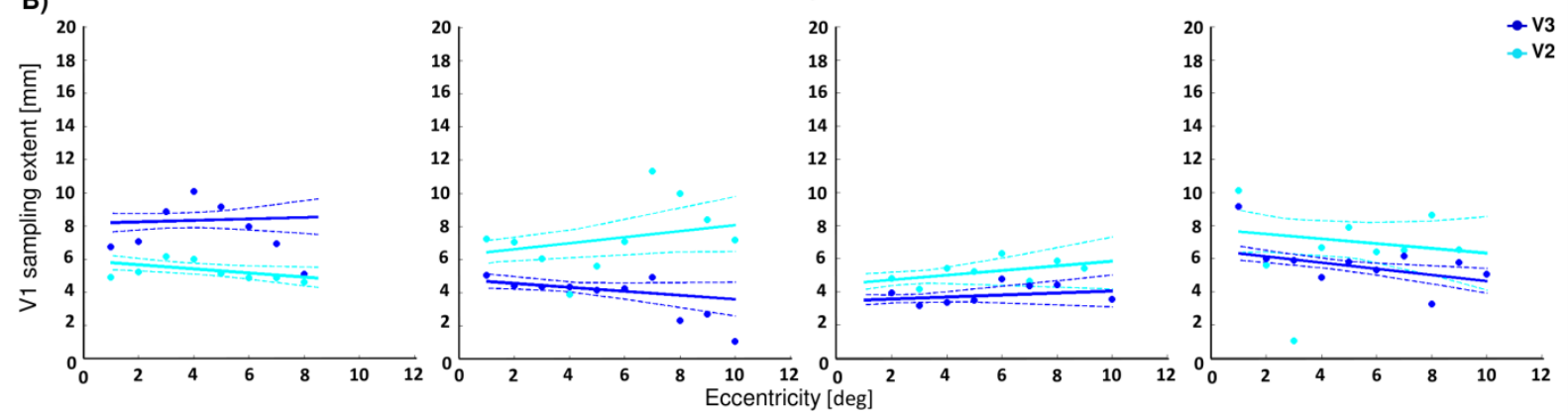

Figure S3: Dependence of $\mathbf{p R F}$ size and CF size on eccentricity. A) Comparison of the pRF 
CHP were restricted to the region with the overlap of all three maps ( $\mathrm{ROI}_{3 \text { maps }}$ ). For both maps from the left eye (middle panels), the pRF sizes increase as a function of eccentricity and through the visual hierarchy (similar to the controls). This expansion through the visual areas is most evident from V1 (black) to V2 (cyan) and V1 to V3 (blue), whereas the difference in pRF sizes of $\mathrm{V} 2$ and V3 is smaller. A similar pattern is observed for the right hemifield map of the right eye (right panel), except for V2 where the pRF size is not increased across visual hierarchy (i.e., V2 pRF size < V1 pRF size). This might be associated with the lower VE and the lower relative activated area in V2 (see Figure 4). B) Comparison of V1-referred CF sizes in V2 (cyan) and V3 (blue) between three maps of CHP (middle and right panels) and the average V1-referred CF size estimates from 4 controls obtained for right hemifield mapping (left panel). The ROIs in CHP were restricted to the region with the overlap of all three maps $\left(\mathrm{ROI}_{3 \mathrm{maps}}\right)$. The $\mathrm{CF}$ size is plotted as a function of eccentricity after adjusting for pRF laterality (Haak et al., 2013). This yields V1 sampling extent which is roughly constant across eccentricities, but increases through the visual hierarchy in the control data. For all three maps of CHP, however, V1 sampling extent in V3 is smaller than that in V2. This alteration might suggest a reduction in spatial coupling between V1 and V3 regions that receive triple hemifield input. Solid lines demonstrate the linear fits for the dots and the dashed lines correspond to the $95 \%$ bootstrapped confidence interval of the linear 844 fits.

A) Even runs; left eye vs right eye

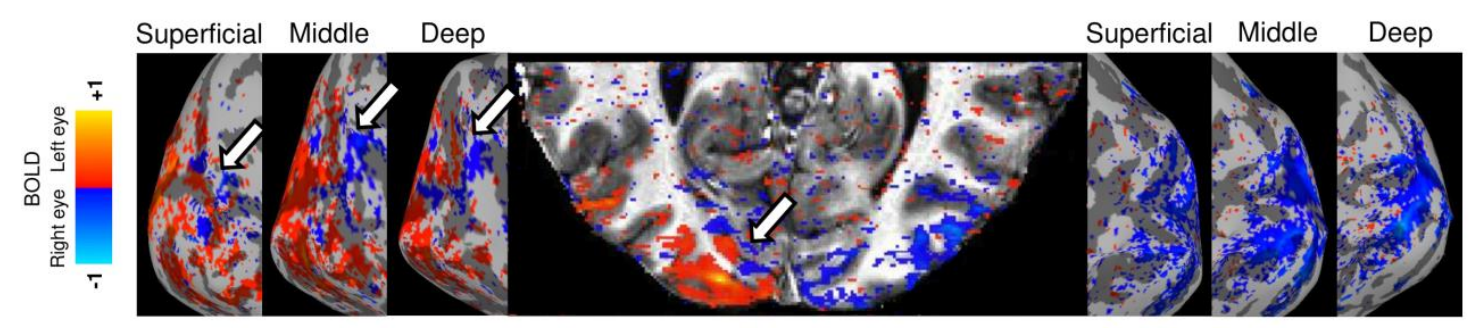

B) Odd runs; left eye vs right eye

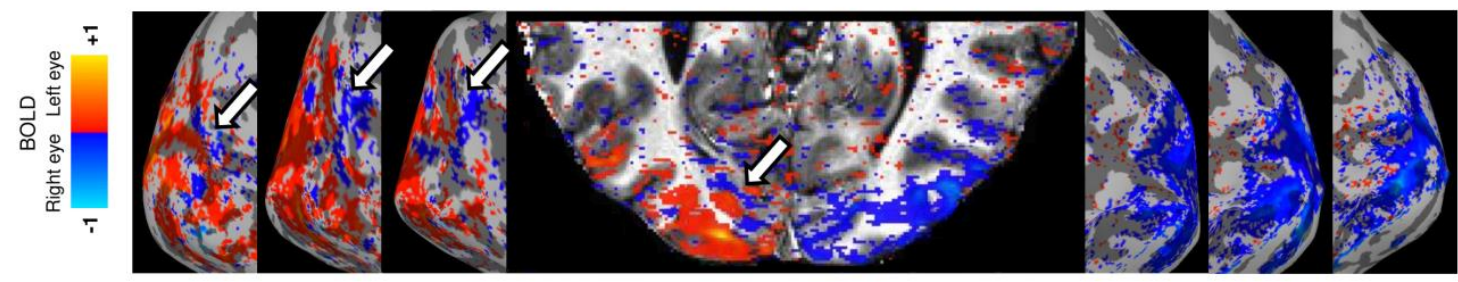

848 Figure S4: Response to left vs right eye stimulation for split data-set (even and odd runs).

849 Similar patterns were observed for both halves of the data, indicating within-session consistency.

850 Conventions as for Figure 6. 